\title{
UNCERTAINTY AND LEARNING IN PHARMACEUTICAL DEMAND
}

\author{
BY GREGORY S. CRAWFORD AND MATTHEW SHUM ${ }^{1}$
}

\begin{abstract}
Exploiting a rich panel data set on anti-ulcer drug prescriptions, we measure the effects of uncertainty and learning in the demand for pharmaceutical drugs. We estimate a dynamic matching model of demand under uncertainty in which patients learn from prescription experience about the effectiveness of alternative drugs. Unlike previous models, we allow drugs to have distinct symptomatic and curative effects, and endogenize treatment length by allowing drug choices to affect patients' underlying probability of recovery. We find that drugs' rankings along these dimensions differ, with high symptomatic effects for drugs with the highest market shares and high curative effects for drugs with the greatest medical efficacy. Our results also indicate that while there is substantial heterogeneity in drug efficacy across patients, learning enables patients and their doctors to dramatically reduce the costs of uncertainty in pharmaceutical markets.
\end{abstract}

KEYWORDS: Uncertainty, learning, pharmaceutical demand, matching, dynamic discrete choice.

\section{INTRODUCTION}

ECONOMISTS HAVE LONG BEEN CONCERNED with the behavioral implications of uncertainty in experience good markets, where utility-bearing characteristics are revealed to consumers through consumption or use. This is particularly true in pharmaceutical markets, where

[d]octor and patient may not know [...] whether a drug is going to be effective or the nature of the side effects. [...] Some people have to cycle through various drugs [...] to find an appropriate match between a drug and their particular problems (Baily (1997, pp. 197-198)).

As this quote illustrates, uncertainty coupled with heterogeneity in patients' illnesses and drugs' effects make any drug-treatment program a complex matching process, requiring time and patience for a doctor to discover the drug which best matches each of his patients' conditions. ${ }^{2}$

In this paper, we measure the effects of uncertainty and learning on prescription choices and treatment outcomes in one large pharmaceutical market: anti-ulcer drugs. We estimate a dynamic matching model of demand under uncertainty in which doctors diagnose a patient's condition and then choose a sequence of drugs to minimize her expected disutility from illness. Doctors (and their patients) are assumed to be uncertain both about the effectiveness

\footnotetext{
${ }^{1}$ We are grateful to the editor and three anonymous referees for their detailed comments, which substantially improved the paper. We also thank Dan Ackerberg, Steve Berry, Donna Gilleskie, Nadia Soboleva, Scott Stern, and seminar participants at Duke, UNC-Chapel Hill, NBER, NYU, Princeton, Queen's, UT-Austin, and Virginia. We thank Andrea Coscelli, Giuseppe Traversa, and Roberto Da Cas for introducing us to the data and acquainting us with features of Italian pharmaceutical markets.

${ }^{2}$ See also Temin (1980) and Arrow (1963) for discussions of the implications of uncertainty for drug choice.
} 
of alternative drugs as well as the probability the patient will remain ill and require additional prescriptions. Each drug impacts each patient differently, and patients learn their idiosyncratic "match values" with each drug through direct prescription experiences. The model is estimated using a rich Italian panel data set that tracks patients' prescriptions for anti-ulcer drugs.

Our modeling of pharmaceutical demand as a dynamic matching process resembles the approach taken in the job-matching literature in labor economics (cf. Jovanovic (1979), Miller (1984)). ${ }^{3}$ Our empirical model builds upon the Bayesian learning models in the empirical dynamic discrete-choice demand literature (cf. Eckstein, Horsky, and Raban (1988), Erdem and Keane (1996), and Ackerberg (2003)). As noted by Erdem and Keane (1996), patient uncertainty can have two competing effects on drug choices. First, if patients are risk-averse, uncertainty about drugs' effectiveness or side effects can lead to persistence in drug choices, as risk aversion makes patients reluctant to try new drugs. On the other hand, if patients are forward-looking, uncertainty encourages consumers to experiment with drugs they have not previously tried in order to gain information that will be useful for making future drug choices. This can lead to diffuse drug choices, counteracting the persistence induced by risk aversion. ${ }^{4}$

This paper extends these literatures in several ways to accommodate features of pharmaceutical markets which distinguish them from other product markets. First, we allow drugs to be differentiated along two dimensions, having both symptomatic effects, which impact a patient's per-period utility directly via symptom relief (or side effects), and curative effects, which impact a patient's probability of recovery. Since patients face uncertainty along both dimensions, the resulting dynamic model features a two-dimensional matching problem. ${ }^{5}$ Second, because the demand for drugs is derived from the demand for good health, we endogenize patients' length of treatment by allowing their drug choices to impact their probability of recovery. This accentuates the importance of dynamic aspects of patient behavior, because a sequence of bad

\footnotetext{
${ }^{3}$ See Eckstein and Wolpin (1987) for a survey of related empirical models.

${ }^{4}$ This paper also joins a growing empirical literature on learning-based models of pharmaceutical demand (Currie and Park (1999), Ching (2002)). We differ from these papers in assuming that patients are forward-looking in their drug choices. There are also a number of theoretical papers that examine how firms may set price and advertising levels to strategically manipulate the consumer learning process (e.g., Nelson (1970), Shapiro (1983), Kihlstrom and Riordan (1984), Milgrom and Roberts (1986), Bergemann and Välimäki (2004)). The latter paper, in particular, considers a dynamic equilibrium monopoly pricing model in which the consumer decision model resembles in many respects the learning model presented in this paper.

${ }^{5}$ Both Erdem and Keane (1996) and Ackerberg (2003) also estimate learning models with two signals (use experience and advertising), but both signals are relevant only for consumers' current utility.
} 
choices not only lowers current utility, but also future utility (by delaying the completion of treatment). ${ }^{6}$

Despite significant "diagnostic matching" by doctors of patients to drugs, our results imply substantial heterogeneity in drugs' match values across patients, highlighting the importance of the matching problem in this market. We find that patients learn quite quickly, however: over two-thirds of patients' initial uncertainty is resolved after a single prescription. Since patients are estimated to be risk-averse, this reduction in uncertainty leads to significant persistence in drug choices after even a single prescription. Counterfactual simulations comparing actual choices to environments that eliminate uncertainty and the ability to experiment demonstrate that learning and experimentation enable patients to achieve levels of discounted utility that are comparable to those available in a "first-best" scenario where uncertainty is removed. Hence, while there is substantial heterogeneity in drug efficacy across patients, we find that learning enables patients and their doctors to dramatically reduce the costs of uncertainty in pharmaceutical markets.

The rest of this paper is organized as follows. In Section 2, we provide some background on pharmaceutical markets, introduce the unique patient-level data set that we employ in our work, and analyze the data for evidence of uncertainty and experimentation. This motivates the economic model and its empirical analog developed in Sections 3 and 4. Section 5 presents estimation results and discusses implications of the estimates, while Section 6 presents counterfactual simulations to measure the costs of uncertainty in the market. Section 7 concludes.

\section{PHARMACEUTICAL MARKETS AND DATA DESCRIPTION}

The available products in any pharmaceutical market are mainly distinguished by their active ingredient (or molecule). While molecules differ in their effectiveness, side effects, or dosage form, competing producers may offer different brands of a given molecule, which differ in price, packaging, and dosage frequency. Since uncertainty is likely to be most important regarding a drug's effectiveness or side effects, we focus on intermolecular choice in the anti-ulcer market. ${ }^{7}$ In what follows, we use the terms "molecule" and "drug" interchangeably.

\footnotetext{
${ }^{6}$ In this respect, our paper also resembles work in the health literature (cf. Gilleskie (1998), Blau and Gilleskie (2000)) in which health outcomes are endogenized and allowed to depend on patients' previous medical and work choices. While neither of these papers allows for learning, see Mira (2000) for a dynamic learning-based model of fertility choice, and Chernew, Gowrisankaran, and Scanlon (2004) for a learning model of health-plan report cards.

${ }^{7}$ In contrast, much of the recent work on the pharmaceutical industry in the United States has explored the nature of intramolecular (i.e., branded vs. generic) competition (e.g., Caves, Whinston, and Hurwitz (1991), Grabowski and Vernon (1992), Scott-Morton (1999), Stern (1996), Frank and Salkever (1997)). In general, this issue does not arise in the Italian market we study because there are no generic manufacturers in this market. Coscelli (2000) is a study of
} 
Despite the potential importance of uncertainty and learning in understanding pharmaceutical demand, there is little empirical evidence of its magnitude or effects. The most closely related pharmaceutical research has focused on measuring the nature of intra- versus intermolecular substitution in pharmaceutical demand using static demand models (Stern (1996), Ellison, Cockburn, Griliches, and Hausman (1997)). Unfortunately, the cross-section patient-level data or panel aggregate (market share) data of the type used in these studies are not rich enough to analyze the importance of uncertainty and learning in fully dynamic models. Since both uncertainty and patient-level heterogeneity in drug effectiveness affect choice, patient-level panel data are required in order to disentangle the confounding effects of uncertainty vs. heterogeneity.

We overcome these constraints in this paper by employing a unique data set of all anti-ulcer prescriptions received by a $10 \%$ sample of patients aged 15-85 in the Rome metropolitan area. ${ }^{8}$ The data are reported monthly over the 36 months between January 1990 and December 1992. About 310,000 observations, which represent prescriptions to over 55,000 patients, are recorded in this data set. Coscelli (2000) describes the data set in more detail.

Two characteristics of the Italian market differentiate it in important ways from the U.S. market. The first is that patients' medical costs are covered by a National Health System, implying that there is no variation in insurance status across patients. This feature of the market mitigates the agency problems associated with heterogeneous third-party payers because doctors face a uniform incentive scheme. ${ }^{9}$ Moreover, the anti-ulcer market is almost entirely drug-based. Agency concerns would appear more acute in therapeutic markets where doctors must often choose between treatment procedures (e.g., surgery, radiation) that are hundreds of times more expensive than drugs.

The top portion of Table I provides a list of the drugs available in the Italian market with market shares greater than $3 \%$. The majority of patients receive Glaxo's ranitidine, marketed in Italy under the brand names Zantac and Ranidil. ${ }^{10}$ Most of the rest of the market is held by Astra's omeprazole (marketed under the brand names Losec and Omeprazen), Merck's famotidine (Pepcid, Famodil), and Lilly's nizatidine (Axid, Zanizal). Since these four molecules account for over $85 \%$ of prescriptions in the Italian anti-ulcer market, we aggregate all but these four leading molecules into a single composite drug, which we refer to as "drug 5 " in what follows.

intramolecular substitution in the Italian anti-ulcer market using the same data employed in this paper.

${ }^{8}$ The drugs in our paper are used to treat many gastrointestinal (GI) conditions. The most common are dyspepsia (i.e., upper-abdominal pain), gastroesophageal reflux disease (GERD), and ulcers. Despite these different indications, we follow the pharmacological literature and refer to them as anti-ulcer drugs.

${ }^{9}$ As such, heterogeneous constraints on doctor choices as induced by health maintenance organization formularies in the U.S. market do not come into play.

${ }^{10}$ Indeed, during the sample period, Zantac was the best-selling drug in the world, with annual sales of over $\$ 25$ billion dollars. 
TABLE I

THE ITALIAN ANTI-UlCER MARKET

\begin{tabular}{|c|c|c|c|c|c|c|c|}
\hline \multicolumn{8}{|c|}{ Summary Statistics from the Data } \\
\hline \# & Molecule & Patent-Holder & $\begin{array}{l}\text { In-Sample } \\
\text { Mkt. Share }^{\mathrm{c}}\end{array}$ & & $\begin{array}{l}\text { 3rands }{ }^{\mathrm{a}} \\
\text { in Mkt. }\end{array}$ & $\begin{array}{l}\text { Date of } \\
\text { Entry }\end{array}$ & $\begin{array}{l}\text { Avg. }{ }^{b} \\
\text { Price }\end{array}$ \\
\hline 1 & Ranitidine & Glaxo & 64.4 & & Ranidil & 1981 & $\$ 2.90$ \\
\hline 2 & Omeprazole & Astra & 11.0 & & meprazen & 1990 & $\$ 3.14$ \\
\hline 3 & Famotidine & Merck & 6.8 & & Famodil & 1986 & $\$ 2.59$ \\
\hline 4 & Nizatidine & Lilly & 3.2 & & Zanizal & 1988 & $\$ 2.74$ \\
\hline 5 & 19 others & - & 14.6 & & ious & $<1981$ & $\$ 1.42^{\mathrm{d}}$ \\
\hline \multicolumn{8}{|c|}{ Overall Statistics } \\
\hline \multicolumn{2}{|c|}{ Variable } & \multicolumn{2}{|c|}{ Num. Obs. } & Mean & Std. Dev. & Min & Max \\
\hline \multicolumn{3}{|c|}{ Number of prescriptions } & 34,972 & 2.82 & 3.87 & 1 & 80 \\
\hline \multicolumn{3}{|c|}{ Number of drugs } & 34,972 & 1.16 & 0.41 & 1 & 5 \\
\hline \multicolumn{3}{|c|}{ Treatment length (mths) } & 34,972 & 5.69 & 7.70 & 1 & 31 \\
\hline \multicolumn{3}{|c|}{ Number of spells } & 34,972 & 1.19 & 0.58 & 1 & 8 \\
\hline \multicolumn{3}{|c|}{ Spell length } & 41,593 & 2.37 & 3.34 & 1 & 72 \\
\hline \multicolumn{3}{|c|}{ Censoring indicator ${ }^{\mathrm{e}}$} & 34,972 & 0.35 & 0.48 & 0 & 1 \\
\hline
\end{tabular}

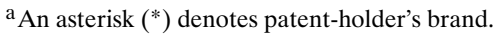

${ }^{b}$ U.S. dollars per daily dose. This was calculated from the reported Italian lire per daily dose using a U.S.-Italian exchange rate (from 1991) of $\$ 1=1,300$ lire.

c Share of total number of prescriptions.

$\mathrm{d}$ Averaged (using share of drug in basket as weight) over all drugs in the composite and averaged over sample period.

${ }^{\mathrm{e}}$ The definition of a censored sequence is described in Section 2.1.
}

Most of the drugs marketed in the late 1970s and the 1980s were based on "H2-receptor antagonists" (hereafter H2As). ${ }^{11}$ Ranitidine, famotidine, and nizatidine (drugs 1, 3, and 4) as well as a majority of the medicines included in the composite drug 5 are based on H2A agents. During the early 1990s, drugs based on "protonic-pump inhibitors" (PPIs) were introduced. These drugs were more effective at treating many GI conditions, but were not (and still are not) approved for others (Yamada (1995)). Among the drugs in our data set, only omeprazole (drug 2) was based on a PPI agent.

Drug prices in the Italian market are set by the regulatory Drug Commission (CUF), following a reference-price scheme in which drugs of therapeutic equivalence - the same active ingredient, the same route of administration, etc.- -are assigned the same price. This limits the extent of price differences between drugs across time, and in our empirical work, we assume that drug prices are constant over time. ${ }^{12}$ Moreover, in the sample period, Italian pa-

\footnotetext{
${ }^{11}$ See Yamada (1995) for an account of the pharmacology of anti-ulcer drugs.

${ }^{12}$ This is a reasonable assumption for drugs $1-4$, as there were little or no changes in the regulated Italian drug prices over the sample period. The average cost of the composite drug 5 does
} 
tients' copayments in the anti-ulcer treatment class were $50 \%$. Hence, in our empirical work, we assume that the price of a drug faced by patients is $50 \%$ of the price reported in the top panel of Table I.

\subsection{Data Description}

Several steps were involved in preparing the data used in this paper. First, since our data do not reveal the order in which a patient received multiple prescriptions within a given month, we exclude all patients who received prescriptions of different drugs within a month. ${ }^{13}$ In addition, for many patients, we do not observe whether they began or ended an ulcer treatment during our sample period. To avoid the difficulties associated with left-censoring as well as ensure homogeneity of patients' choice sets over time, we include only patients who are first observed after the sixth month of the sample (i.e., June 1990). ${ }^{14}$ This month was chosen since it marked the entry of drugs based on the molecule omeprazole into the Italian market. Together, these filters eliminated 14,670 patients from the original sample, leaving us with 34,972 patients (and a total of 98,634 prescription episodes). Furthermore, we define a prescription sequence as right-censored if the last in-sample prescription fell within the last 6 months of the sample period. Such a long window was used because large gaps of several months or more between prescriptions were not uncommon in the data set. As the bottom panel of Table I shows, roughly one-third of the prescription sequences are classified as censored.

Patients receive, on average, 2.8 prescriptions for 1.2 drugs over a period of just under 6 months. ${ }^{15}$ These averages mask important differences in the intensity of treatment across patients, however. Figure 1 plots the empirical distributions of several key variables for the patients in our sample. Clearly, the number of prescriptions among the patients in the data set is very skewed: $54.1 \%$ of patients in the sample receive a single prescription, $17.1 \%$ receive two, and only $3.9 \%$ receive ten or more. Despite this, the latter group accounts for $26.2 \%$ of total prescriptions written.

Previous research (e.g., Hellerstein (1997), Coscelli (2000)) has documented significant persistence in drug choices among patients and their doctors, and these data are no exception. The repeat purchase probability (measured as

change over time as the market shares of its various components change, but this change is sufficiently small that a constant price is not a bad approximation.

${ }^{13}$ In principle, these data could be included and (the probability distribution of) their likely order estimated. However, such a procedure quickly becomes computationally costly when a patient has multiple periods in which she takes more than one drug, so it is not attempted here.

${ }^{14}$ Ackerberg and Rysman (2003) discuss the dangers of exploiting variation in choice sets for demand estimation in static discrete-choice models.

${ }^{15}$ In this section, treatment length is defined for each patient as the month of the last prescription received in the sample less the month of the first prescription plus 1 . In the estimation and applications, treatment length refers to the number of prescriptions taken. 

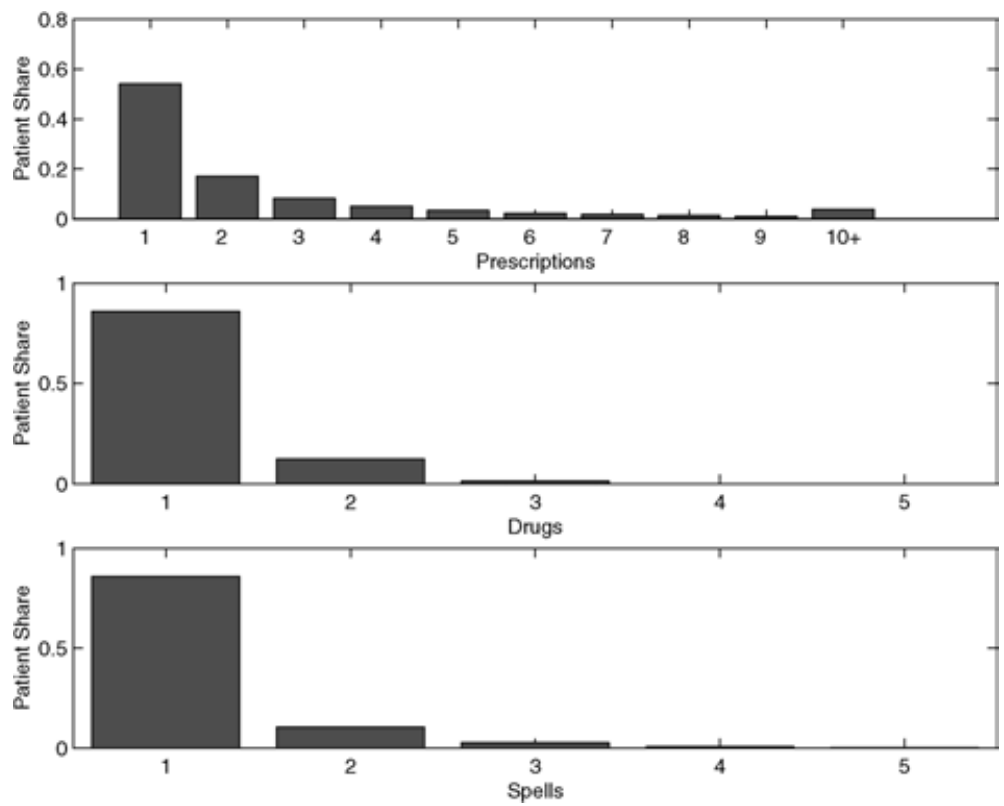

FIGURE 1.-Histogram of prescriptions, drugs, and spells.

the share of patients who took the same drug in period $t$ that they took in period $t-1$ ) ranges from a low of $74.8 \%$ for the composite drug 5 to a high of $94.1 \%$ for the market leader, ranitidine.

We next consider evidence of learning and experimentation in our raw data. We first examine the incidence of switching between drugs. We define a drug "spell" to be a sequence of one or more prescriptions to a single drug. The bottom panel of Table I and Figure 1 show that patients have an average of 1.19 spells, but this distribution is extremely skewed among the patients in the sample. The average spell consists of 2.37 prescriptions, decreasing only slightly to 2.08 prescriptions for spells that end with the choice of another drug. This suggests that it takes some time for patients to learn if a drug is working for them.

Next, in Table II, we compute the average probability (i.e., frequency) of switching drugs at different junctures during a treatment. ${ }^{16}$ Since switching may be induced by both experimentation (which is strongest when patients are ill-informed) and information (after consumers have learned that their match value with a given drug is low), we look for evidence of experimentation at the beginning of treatments, when consumers have the least information (and thereby the incentive to experiment is strongest).

\footnotetext{
${ }^{16}$ To avoid interpretational ambiguities due to censoring of the prescription sequences, we use only the uncensored observations to compute the figures in this table.
} 
TABLE II

SWitching Probabilities over the Course of TreatmenT ${ }^{\mathrm{a}}$

\begin{tabular}{lrrrrrr}
\hline \hline \multirow{2}{*}{$\begin{array}{l}\text { Prescription } \\
\text { Number }\end{array}$} & \multicolumn{6}{c}{ Total Treatment Length } \\
\cline { 2 - 7 } & \multicolumn{1}{c}{5} & \multicolumn{1}{c}{6} & \multicolumn{1}{c}{7} & \multicolumn{1}{c}{8} & 9 & 10 \\
\hline 2 & 14.3 & 13.6 & 10.9 & 10.0 & 7.8 & 9.2 \\
3 & 11.6 & 11.6 & 6.3 & 8.8 & 7.8 & 6.6 \\
4 & 8.9 & 5.6 & 5.4 & 3.1 & 7.8 & 3.9 \\
5 & 13.4 & 7.9 & 10.0 & 8.8 & 4.9 & 5.3 \\
6 & & 11.3 & 6.3 & 5.7 & 2.9 & 5.3 \\
7 & & & 9.5 & 10.0 & 7.8 & 11.8 \\
8 & & & & 8.1 & 4.9 & 11.8 \\
9 & & & & & 7.8 & 5.3 \\
10 & & & & & & 11.8 \\
\hline
\end{tabular}

${ }^{\text {a }}$ The $(i, j)$ th entry is the percentage of treatment sequences of length $j$ in which a switch was observed during the $i$ th $(i \leq j)$ prescription.

We see that across several treatment lengths and spell transitions, there is a marked decreasing trend in the switching probability at the very beginning of treatment. For example, for patients with an eight-prescription treatment length, the probability of switching is $10.0 \%$ after the first prescription, then falls to $8.8 \%$ after the second prescription, and falls further to $3.1 \% .^{17}$ As treatment proceeds beyond the fourth prescription, however, the switching probabilities start to rise. While merely suggestive, higher switching probabilities after early prescriptions are consistent with experimentation, while higher switching probabilities after late prescriptions are consistent with learning. More definitive statements require a model of pharmaceutical choice under uncertainty that can disentangle these effects.

\section{A BIVARIATE BAYESIAN LEARNING MODEL OF PHARMACEUTICAL DEMAND}

\subsection{Overview}

The model introduced below is a matching model of drug choice. We assume patients come to their doctor to seek treatment for an unknown gastrointestinal illness. ${ }^{18}$ Upon arrival, the doctor performs an initial diagnostic of the patient to assess the severity of her illness. Based on this initial diagnosis and his resulting beliefs about the efficacy of alternative drugs, the doctor selects an initial drug treatment. The patient takes this drug and, if not immediately healed, reports back to the doctor its impact on her condition. Further prescriptions follow— to the same or different drugs—until the patient is healed.

\footnotetext{
${ }^{17}$ Similar patterns arise if one considers only transitions from the first spell.

${ }^{18}$ While there are a number of related conditions treated by the drugs we study, for simplicity we will refer to all of them as ulcers and to this class of drugs as anti-ulcer drugs.
} 
Conditional on the initial diagnosis, each doctor (and his patient) is assumed to have uncertainty about the effectiveness of various drug alternatives for that patient. This uncertainty is modeled by patient-specific "match values" (which are unknown at the beginning of treatment) associated with each drug in the market. A given drug's match values can differ across patients, thereby accommodating heterogeneity in drug effectiveness across patients. In the bivariate learning model considered in this paper, we allow patients to have two separate match values for each drug, corresponding to the two important dimensions of differentiation among the choices in many drug (and medical) markets. First, a drug's symptomatic effect measures its impact to patient utility from symptom relief and/or side effects and, second, its curative effect measures its impact to a patient's probability of recovery.

Each patient (and her doctor) learns about her two match values through the experience signals coming from direct use of alternative drugs. Doctors have prior beliefs about these match values that differ depending on the outcome of the patient's initial diagnosis as well as the doctors idiosyncratic experience with the various drugs. The prescription experience signals for each patient, in turn, are assumed to yield noisy signals about their match values. Whereas utility is a function of experience signals and these signals depend on the match values, patients have an incentive to learn their match values for each drug. An optimal sequence of drug choices is then obtained as the solution to a dynamic Bayesian learning model.

Each doctor is forward-looking and selects the sequence of drugs that maximizes his patient's (present-discounted) expected utility,

$$
\max _{D \equiv\left\{\left\{d_{j n t} t_{n=1}^{N}\right\}_{t=1}^{\infty \infty}\right.} E_{D} \sum_{t=1}^{\infty} \beta^{t} d_{j n t} u_{j n t}\left(1-w_{j, t-1}\right)
$$

where $d_{j n t}$ is an indicator that equals 1 if patient $j$ takes drug $n$ in period $t$, $u_{j n t}$ measures the single-period utility flow to patient $j$ from taking drug $n$ in period $t, w_{j t}$ is an indicator for whether patient $j$ recovers after period $t$, and $\beta \in[0,1]$ is a discount factor. ${ }^{19}$ The expectation is taken over the distribution of future utilities and treatment lengths induced by the policy sequence $D$. The doctor for patient $j$ maximizes (1) by choosing the sequence $D$, which specifies, in each period $t$ (for $t=1, \ldots, \infty$ ), the drug $n$ that should be tried. ${ }^{20}$

There are two sources of patient-level uncertainty in the model. First, even given the initial diagnosis, a doctor does not know his patients' drug-specific

\footnotetext{
${ }^{19}$ In our empirical work below, $\beta$ is not estimated, but rather fixed at a value of 0.95 .

${ }^{20}$ Drug choice is only one part of the treatment process, which also includes the decision to go see a doctor and being prescribed drugs (versus some other treatment). Since our data set is silent as to either the incidence of gastrointestinal conditions in the population or the incidence of doctor visits resulting in a prescription, our results necessarily apply only to the population of ulcer sufferers who obtain (and fill) a prescription for a drug therapy, and should be interpreted with that caveat.
} 
match values and learns about these values only gradually over time, as his patient receives experience signals from the drugs that he prescribes. For patient $j$, each drug $n$ is characterized by two time-invariant match values, $\mu_{j n}$ and $\nu_{j n}$, which summarize, respectively, the symptomatic and curative effects of the drug for that patient. Subsequently, each prescription yields two separate signals, corresponding to these two different match values. The symptomatic signal $x_{j n t}$, which patient $j$ derives from taking drug $n$ during period $t$, is drawn from a distribution with the symptomatic match value $\mu_{j n}$ as the unknown mean. The curative signal $y_{j n t}$ is likewise drawn from a distribution centered around the curative match value $\nu_{j n}$.

Second, a doctor does not know his patient's length of treatment. After each prescription, patient $j$ has some probability of recovering, requiring no further prescriptions. The probability that patient $j$ recovers by the end of period $t$ can potentially depend on all the curative signals that patient $j$ has obtained from her previous drugs, up to (and including) period $t$. As patients take alternative drugs, they learn about the symptomatic and curative effectiveness of each. Prescriptions continue until the patient recovers (i.e., $w_{j t}=1$ ).

\section{On patients and doctors}

The focus of this paper is the therapeutic uncertainty facing patients and their doctors about the effectiveness of pharmaceutical drugs for a given patient. An important related issue in drug markets is the therapeutic uncertainty facing doctors about the (average) effectiveness of drugs across patients. ${ }^{21}$ Our patient-level data do not permit us to directly analyze learning by doctors across patients. We do, however, accommodate its effects for omeprazole, the innovative entrant in this market, by allowing doctors' beliefs for omeprazole to evolve over time. This is similar to the approach taken by Ching (2002) in the market for cardiovascular, antidepressant, and antibiotic drugs, and is empirically important in our application. ${ }^{22}$

We also assume that doctors are selecting drugs exclusively to maximize the utility of their patients, in effect making doctors and patients a single decisionmaker. This abstracts away from important agency problems that can arise due

\footnotetext{
${ }^{21}$ While it has long been recognized that many factors, including direct experience, results of clinical trials, and promotional material provided by drug makers, influence doctors' prescription patterns, the growing field of pharmacogenetics, or personalized medicine, is analyzing the impact of a patient's genetic makeup on the incidence and severity of various illnesses and the effectiveness of alternative drug therapies. It is widely believed that the long-term future of the pharmaceutical industry will depend on the effective development of personalized medicines (Reuters (2004), Arnst (2004)). Until diagnostic procedures able to identify genetic responsiveness to drugs are developed, however, both types of learning are likely to be important in pharmaceutical markets.

${ }^{22}$ See also Coscelli and Shum (2004) and Ferreyra (1999) for empirical models of doctor-level learning.
} 
to informational asymmetries between doctors and patients that influence doctors' decisions about the drugs to prescribe and are not directly related to a given patient's medical condition. ${ }^{23}$ Of particular interest in the economic literature are the impact of insurance status and out-of-pocket expenditure on drug choices (cf. Mott and Kreling (1998), Iizuka (2004)).

The assumption that doctors are perfect agents for their patients is a strong one and has important implications for our model of drug choice. In particular, perfect agency rules out the possibility of "doctor effects" conditional on a patient's diagnosis: all doctors in our model have the same probability of prescribing a given drug to a patient with a given diagnosis in a given time period. If doctors face the same distribution of diagnoses, then the probability distribution for their prescribed drugs should be identical. In our model, the only way that doctor effects would appear is in an unconditional sense, i.e., if different doctors face different distributions of diagnoses.

While some of the institutional features of the Italian pharmaceutical market described in Section 2 might mitigate concerns about agency issues, we would of course like to allow for and estimate any relevant doctor effects. Unfortunately, our data do not provide sufficient doctor-level information to do so. ${ }^{24}$ Our results should therefore be interpreted with this caveat in mind, leaving the analysis of agency issues in pharmaceutical markets as an important topic for further research.

\subsection{Model Details}

\section{Diagnostic matching and patient types}

Prior to writing any prescriptions, we assume doctors engage in the "diagnostic matching" of patients to drugs. In particular, we assume that doctors make an initial diagnosis of the type and severity of a patient's illness. Given this diagnosis, we assume they then prescribe one of the available drugs that they feel will best treat the patient's condition. While a patient's diagnosis and her doctor's beliefs are known to each patient and her doctor, they are not known to the econometrician and are therefore a source of unobserved heterogeneity. This is an important problem in our setting, because failing to accurately capture doctor beliefs at the time of the initial prescription can bias our estimates of the degree and consequences of patient-level uncertainty in drug choices.

\footnotetext{
${ }^{23}$ See Mott, Schommer, Doucette, and Kreling (1998) for a survey of relevant agency relationships in pharmaceutical markets and Stern and Trajtenberg (1998) for a recent empirical examination of the possible importance of this issue.

${ }^{24}$ We estimated a model that allows for doctor-specific random effects where the distribution of these effects depended on limited presample doctor prescription histories. We found very little evidence of doctor effects. A full treatment of the question, however, would ideally include full prescription histories and information on exposure to pharmaceutical marketing (e.g., detailing visits, free samples received, etc.) for each doctor in the data, something we do not observe.
} 
To address this problem, we allow doctors to diagnose that a patient's condition falls into one of $K$ types. These patient types influence the model in two ways. First, because patients with different conditions may differ in the underlying severity of their illness, we allow patient types to differ in their initial probability of being healed. In particular, let $h_{0 j}$ denote the probability that patient $j$ can be healed without any treatment at all. We refer to $h_{0 j}$ as patient $j$ 's "initial illness severity" and assume that patient $j$ 's doctor is able to determine $h_{0 j}$ at the initial diagnosis. ${ }^{25}$ In our empirical work, we assume that $h_{0 j}$ takes one of $K$ values,

$$
h_{0 j}=\theta_{k} \quad \text { w.p. } p_{k}, \quad k=1, \ldots, K,
$$

where $0<p_{i}<1, \sum p_{i}=1$, and $0 \leq \theta_{1}, \ldots, \theta_{K} \leq 1$. In what follows, we refer to patients with an initial illness severity $\theta_{i}$ as Type $i$ patients, for $i=1, \ldots, K$.

Second, because patients with different conditions are likely to react differently to the various available drugs, we allow their doctors' prior beliefs regarding the symptomatic and curative match values to differ across patient types.

\section{Preferences}

Patient $j$ 's single-period utility from drug $n$ in time period $t, u_{j n t}$, is assumed to be a function of $x_{j n t}$, patient $j$ 's symptomatic signal from taking drug $n$ in period $t$, and the per-prescription price of drug $n, p_{n}$. Given our focus on uncertainty, the usual linear utility specifications used in the discrete-choice demand literature are too restrictive because the risk neutrality embodied in such a specification removes many of the distinctive effects of uncertainty on the dynamics of individual decision-making. As noted by Erdem and Keane (1996), it is difficult to generate the substantial persistence in choices without risk aversion.

Therefore, we consider a quasilinear utility specification, additively separable in a concave "subutility" function of the drug return and a linear term in price. We assume a Constant Absolute Risk Aversion (exponential) specification for the subutility function, yielding

$$
u\left(x_{j n t}, p_{n}, \epsilon_{j n t}\right)=-\exp \left(-r * x_{j n t}\right)-\alpha * p_{n}+\epsilon_{j n t},
$$

where $r(>0)$ measures the degree of risk aversion and $\epsilon_{j n t}$ is an additive idiosyncratic error that measures idiosyncratic tastes for drug $n$ by patient $j$ in period $t .{ }^{26}$

\footnotetext{
${ }^{25}$ This reflects reasonably well the nature of the diagnosis process: the presence and severity of an ulcer is relatively easy to diagnose (via patient interviews, stomach x-rays, and/or endoscopies), so that uncertainty regarding illness severity is likely to be resolved during the initial diagnosis, while uncertainty regarding a patient's specific reaction to a specific drug is resolved only gradually over time via the patient's direct prescription experiences with the drug.

${ }^{26}$ Moreover, up to the idiosyncratic error $\epsilon_{j n t}$, the per-period utility derived from drug use is negative. This is appropriate because we normalize the terminal utility associated with being
} 


\section{Recovery probabilities}

Let $h_{j t}$ denote the probability that patient $j$ recovers by the end of period $t$ (after she has taken a drug in period $t$ ). We assume that the sequence of recovery probabilities for this patient evolve as

$$
h_{j t}\left(h_{j t-1}, y_{j n t}\right)=\frac{\left(\frac{h_{j t-1}}{1-h_{j t-1}}\right)+d_{j n t} y_{j n t}}{1+\left(\left(\frac{h_{j t-1}}{1-h_{j t-1}}\right)+d_{j n t} y_{j n t}\right)}
$$

with the initial condition $h_{0 j}$ as defined in (2). ${ }^{27}$ As we remarked before, we assume that the illness severity parameters, $h_{0 j}$, are observed by the patient and her doctor, but not by the researcher, and is thus a source of unobserved heterogeneity. Furthermore, since $h_{j t}$ depends on $y_{j n t}$, the curative signal for the drug prescribed to patient $j$ in period $t, h_{j t}$ is random when patient $j$ is deciding which drug to take in period $t$.

\subsection{The Learning Process}

Doctors are assumed to begin treatment with prior information about their patient's two match values, $\mu_{j n}$ and $\nu_{j n}$. Each doctor's prior beliefs about these idiosyncratic match values are summarized by a multivariate normal distribu$\operatorname{tion}^{28}$

$$
\left(\begin{array}{c}
\mu_{j n} \\
\nu_{j n}
\end{array}\right) \sim N\left(\left[\begin{array}{c}
\underline{\mu}_{n k} \\
\underline{\nu}_{n k}
\end{array}\right], \quad\left[\begin{array}{cc}
\underline{\sigma}_{n}^{2} & 0 \\
0 & \underline{\tau}_{n}^{2}
\end{array}\right]\right)
$$

In this expression, $\mu_{n}$ and $\underline{\nu}_{n}$ denote the prior mean match values of drug $n$ and the standard deviations $\left(\underline{\sigma}_{n}, \underline{\tau}_{n}\right)$ are measures of the accuracy of these prior means. In (5), the $k$ subscript denotes the illness severity type of patient $j$ (cf. (2) and the discussion around it).

While patient $j$ does not know $\mu_{j n}$ and $\nu_{j n}$ as she commences treatment, she receives prescription signals which allow her to update regarding these

healed to zero. With a zero terminal value, if utilities were positive, then patients in our model may rationally delay recovery as long as possible in order to continue receiving positive utility from drug use. Such a motive appears implausible for pharmaceutical (or health) markets.

${ }^{27}$ Since signals can potentially be negative, if a particular realization of $y_{j n t}$ becomes too large and negative, the expression in (4) will no longer be a valid probability. While it is difficult to restrict the parameter values explicitly so that this does not happen, we confirm that this does not happen for each set of parameter estimates that we report below.

${ }^{28}$ Estimating the bivariate learning model is computationally costly due to the large number of state variables. As such, we do not explore the possibility that either signals or priors for drugs' symptomatic and curative match values are correlated. See Ackerberg (2003) for a related discussion of the computational difficulties associated with a two-signal model with correlation in the priors. 
unknown quantities. The symptomatic and curative signals are assumed to be independent and distributed normally across periods, ${ }^{29}$

$$
\left(\begin{array}{c}
x_{j n t} \\
y_{j n t}
\end{array}\right) \sim N\left(\left[\begin{array}{c}
\mu_{j n} \\
\nu_{j n}
\end{array}\right], \quad\left[\begin{array}{cc}
\sigma_{n}^{2} & 0 \\
0 & \tau_{n}^{2}
\end{array}\right]\right)
$$

where $\left(\mu_{j n}, \nu_{j n}\right)$ are patient $j$ 's unknown match values for drug $n$ (i.e., the means of the distribution of patient $j$ 's symptomatic and curative signals for drug $n)$ and $\left(\sigma_{n}^{2}, \tau_{n}^{2}\right)$ measure the variation in, respectively, the symptomatic and curative signals around their mean.

We assume that doctors have rational expectations, so that their prior beliefs correspond to the actual distribution of the idiosyncratic match values $\left(\mu_{j n}, \nu_{j n}\right)$ for each drug $n$ across patients $j$ and types $k$ in the market. Accordingly, an alternative interpretation of these prior beliefs is that $\underline{\mu}_{n k}$ (resp. $\left.\underline{\nu}_{n k}\right)$ is the population mean symptomatic (resp. curative) match value of drug $n$ and $\underline{\sigma}_{n}$ (resp. $\underline{\tau}_{n}$ ) measures the heterogeneity or dispersion in symptomatic (resp. curative) match values across patients of type $k$. The values $\underline{\mu}_{n k}, \underline{\nu}_{n k}$ $(k=1, \ldots, K), \sigma_{n}, \tau_{n}, \underline{\sigma}_{n}$, and $\underline{\tau}_{n}$ are all assumed to be known by the patient (or her doctor), and are parameters to be estimated.

The rational expectations assumption is less attractive for drug 2, omeprazole, which entered at the beginning of our sample period. We therefore allowed the prior means $\mu_{2 k}$ and $\underline{\nu}_{2 k}$ to vary across time, and take different values in 6-month intervals (i.e., Jun-Dec 1990, Jan-Jun 1991, Jul-Dec 1991, Jan-Jun 1992, and Jul-Dec 1992). We imposed rational expectations only in the final half-year period, so that we allow doctors' beliefs concerning omeprazole to differ from the true distribution of match values in the sample period before July 1992. In this way, we accommodate learning regarding omeprazole's efficacy that is common across doctors as might arise from attendance at medical conferences and/or publication in leading medical journals.

Letting $l_{j n}^{t}$ denote the number of times that patient $j$ has taken drug $n$ up to (and including) period $t$ and defining the initial values $\mu_{j n}^{0}=\underline{\mu}_{n k}$ and $V_{j n}^{0}=\underline{\sigma}_{n}$, patient $j$ 's posterior beliefs regarding her symptomatic match value $\mu_{j n}$ are given recursively by the sequence of normal distributions with the following

\footnotetext{
${ }^{29}$ The normality assumptions imply that the posterior beliefs on the match values at any point in time follow a normal distribution characterized by two scalar parameters: the posterior mean and the number of signals received to date, which are the state variables in the dynamic optimization model. Without normality, the posterior distributions themselves are the (infinitedimensional) state variables of the dynamic optimization problem, leading to difficulties in computation and estimation.
} 
mean and variance (see DeGroot (1970, Chap. 9)):

$$
\begin{aligned}
& \mu_{j n}^{t+1}=\left\{\begin{array}{l}
\frac{\frac{\mu_{j n}^{t}}{V_{j n}^{t}}+\frac{x_{j n t+1}}{\sigma_{n}^{2}}}{\frac{1}{V_{j n}^{t}}+\frac{1}{\sigma_{n}^{2}}}=\frac{\sigma_{n}^{2} \mu_{j n}^{t}+V_{j n}^{t} x_{j n t+1}}{\sigma_{n}^{2}+V_{j n}^{t}}, \\
\quad \text { if drug } n \text { taken in period } t+1, \\
\mu_{j n}^{t}, \quad \text { otherwise, }
\end{array}\right. \\
& V_{j n}^{t+1}= \begin{cases}\frac{1}{\frac{1}{\sigma_{n}^{2}}+\frac{l_{n}^{t+1}}{\sigma_{n}^{2}}}=\frac{\sigma_{n}^{2} \underline{\sigma}_{n}^{2}}{\sigma_{n}^{2}+l_{j n}^{t+1}{\underline{\sigma_{n}^{2}}}_{n}^{2}}, & \text { if drug } n \text { taken in period } t+1, \\
V_{j n}^{t}, & \text { otherwise. }\end{cases}
\end{aligned}
$$

The analogous expressions for the posterior beliefs on the curative match value are

$$
\begin{aligned}
& \nu_{j n}^{t+1}= \begin{cases}\frac{\tau_{n}^{2} \nu_{j n}^{t}+R_{j n}^{t} y_{j n t+1}}{\tau_{n}^{2}+R_{j n}^{t}}, & \text { if drug } n \text { taken in period } t+1, \\
\nu_{j n}^{t}, & \text { otherwise, }\end{cases} \\
& R_{j n}^{t+1}= \begin{cases}\frac{\tau_{n}^{2} \underline{\tau}_{n}^{2}}{\tau_{n}^{2}+l_{j n}^{t+1} \underline{\tau}_{n}^{2}}, & \text { if drug } n \text { taken in period } t+1, \\
R_{j n}^{t}, & \text { otherwise },\end{cases}
\end{aligned}
$$

where $\nu_{j n}^{t}$ and $R_{j n}^{t}$ are the mean and variance of the normal distribution that characterizes patient $j$ 's beliefs at prescription $t$, with initial conditions $\nu_{j n}^{0}=\underline{\nu}_{n k}$ and $R_{j n}^{0}=\underline{\tau}_{n}$.

\subsection{Dynamic Drug Choice}

In our bivariate dynamic learning model, there are four categories of state variables. The first are patient $j$ 's posterior mean match values $\mu_{j n}^{t}$ and $\nu_{j n}^{t}$, for drugs $n=1, \ldots, 5$. The second are counts of the number of times that patient $j$ has tried each drug, i.e., $l_{j n}^{t}, n=1, \ldots, 5$. The third state variable is $h_{j t}$, the recovery probability for patient $j$ at the end of period $t$. Finally, there are the idiosyncratic errors $\epsilon_{j n t}, n=1, \ldots, 5$. We use $\mathcal{S}_{t}$ to denote the vector of state variables $\left(\mu_{j 1}^{t}, \ldots, \mu_{j 5}^{t}, \nu_{j 1}^{t}, \ldots, \nu_{j 5}^{t}, l_{j 1}^{t}, \ldots, l_{j 5}^{t}, h_{j t}, \epsilon_{j 1 t}, \ldots, \epsilon_{j 5 t}\right)$ for period $t$.

The transition rules for all the state variables can be written in Markovian form. The rules for the posterior means $\mu_{j n}^{t}$ and $\nu_{j n}^{t}$ are given in the first row 
of (7) and the transition rules for the $l_{j n}^{t}$ 's are simply

$$
l_{j n}^{t+1}= \begin{cases}l_{j n}^{t}+1, & \text { if drug } n \text { taken in period } t \\ l_{j n}^{t}, & \text { otherwise. }\end{cases}
$$

Finally, the transition rule for the recovery probability, $h_{j t}$, in the baseline model is given by (4).

\section{Dynamic decision-making}

The value function $W\left(\mathcal{S}_{t}\right)$ in the infinite-horizon problem defined by (1) is recursively defined via Bellman's equation,

$$
\begin{aligned}
& W\left(\mathcal{S}_{t}\right)=\max _{n} E[ u\left(x_{j n t}, p_{n}, \epsilon_{j n t}\right) \\
&\left.+\beta\left(1-w_{j t}\right) E\left[W\left(\mathcal{S}_{t+1}\right) \mid x_{j n t}, y_{j n t}, n\right] \mid \mathcal{S}_{t}\right] \\
&=\max _{n} E[ u\left(x_{j n t}, p_{n}, \epsilon_{j n t}\right) \\
&\left.+\beta\left(1-E\left[w_{j t} \mid y_{j n t}\right]\right) E\left[W\left(\mathcal{S}_{t+1}\right) \mid x_{j n t}, y_{j n t}, n\right] \mid \mathcal{S}_{t}\right] \\
&=\max _{n} E\left[u\left(x_{j n t}, p_{n}, \epsilon_{j n t}\right)\right.\left.\quad+\beta\left(1-h_{j t}\left(h_{j t-1}, y_{j n t}\right)\right) E\left[W\left(\mathcal{S}_{t+1}\right) \mid x_{j n t}, y_{j n t}, n\right] \mid \mathcal{S}_{t}\right] \\
&=\max _{n}\left\{-\exp \left(-r \mu_{j n}^{t}+\frac{1}{2} r^{2}\left(\sigma_{n}^{2}+V_{j n}^{t}\right)\right)-\alpha p_{n}+\epsilon_{j n t}\right. \\
&\left.\quad+\beta E\left[\left(1-h_{j t}\left(h_{j t-1}, y_{j n t}\right)\right) E\left[W\left(\mathcal{S}_{t+1}\right) \mid x_{j n t}, y_{j n t}, n\right] \mid \mathcal{S}_{t}\right]\right\} \\
& \equiv \max _{n}\left\{W_{n}\left(\mathcal{S}_{t}\right)\right\},
\end{aligned}
$$

where the fourth equality follows from the exponential form of the per-period utility function and the foregoing distributional assumptions made on the priors and signals. ${ }^{30}$ In the above, $\mathcal{S}_{t+1}$ denotes the values of the state variables in period $t+1$ and $W_{n}\left(\mathcal{S}_{t}\right)$ denotes the choice-specific value function for drug $n$.

${ }^{30}$ That is,

$$
\begin{aligned}
E_{x_{j n t}} u_{j n t} & =-E_{\mu} E_{x \mid \mu} \exp \left(-r x_{j n t}\right)-\alpha * p_{n}+\boldsymbol{\epsilon}_{j n t} \\
& =-E_{\mu} \exp \left(-r \mu_{j n}+\frac{1}{2} r^{2} \sigma_{n}^{2}\right)-\alpha * p_{n}+\epsilon_{j n t} \\
& =-\exp \left(-r \mu_{j n}^{t}+\frac{1}{2} r^{2}\left(\sigma_{n}^{2}+V_{j n}^{t}\right)\right)-\alpha * p_{n}+\epsilon_{j n t} .
\end{aligned}
$$


Hence, the optimal policy takes a very simple form: in each period $t$, patient $j$ should choose the drug $n$ with the highest value function $W_{n}\left(\mathcal{S}_{t}\right)$. The term $\frac{1}{2} r^{2}\left(\sigma_{n}^{2}+V_{j n}^{t}\right)$ represents the "risk premium," the disutility incurred by patient $j$ due to the uncertainty she faces regarding both her match value $\mu_{j n}$ as well as the drug return $x_{j n t}$. It is clear, from the equations above, that risk aversion generates persistence in drug choice via the risk premium. ${ }^{31}$ Patient $j$ 's previous use of drug $n$ reduces the posterior variance $V_{j n}^{t}$ and, therefore, increases the expected utility of drug $n$ relative to drugs that patient $j$ has not previously used. This, in turn, increases the probability that patient $j$ chooses drug $n$ again. Indeed, the risk premium functions as a "switching cost," by making patient $j$ reluctant to choose drugs that she has not tried before (and for which the variance $V_{j n}^{t}$ is high).

Assuming stationarity of the optimal policy and letting $\vec{x}_{j n} \equiv\left(x_{j n}, y_{j n}\right)^{\prime}$ denote the vector of symptomatic and curative signals obtained from a prescription of drug $n$, the Bellman equation becomes

$$
\begin{aligned}
W(\mathcal{S})=\max _{n} E\{ & u\left(x_{j n}, p_{n}, \epsilon_{j n t}\right) \\
& \left.+\beta\left(1-h_{j}^{\prime}\left(h_{j}, y_{j n}\right)\right) E\left[W\left(\mathcal{S}^{\prime}\right) \mid \vec{x}_{j n}, n\right] \mid \mathcal{S}\right\},
\end{aligned}
$$

where the primes (') denote next-period values. A number of researchers have surveyed the available methods for computing the value function; see, for example, Judd (1998, Chap. 12) or Rust (1996). We employ a variant of the Keane and Wolpin (1994) approximation method for computing the value function; we describe it in more detail in the Appendix.

\section{THE ECONOMETRIC MODEL}

For each patient $j$, our data yield observations on the sequence of drugs chosen, $d_{j 1 t}, \ldots, d_{j N t}$, as well as the treatment length $T_{j}$. Let $I_{j}$ denote a censoring indicator for whether patient $j$ 's treatment length is censored by the end of the sample period (i.e., if $I_{j}=1$, then $T_{j}$ is a lower bound on patient $j$ 's actual treatment length).

Define $W_{j n t} \equiv W_{n}\left(\mathcal{S}_{t}\right)$ as patient $j$ 's value function for drug $n$ during period $t$ and let $\vec{x}_{j n t, k} \equiv\left\{x_{j n 1, k}, \ldots, x_{j n t, k}, y_{j n 1, k}, \ldots, y_{j n t, k}\right\}$ denote the experience signals received by patient $j$ up to (and including) period $t$. Note that her initial healing probability, $h_{0 j, k}$, and these experience signals depend on the patient's

\footnotetext{
${ }^{31}$ As is clear from (9), the parameter $r$ measures both the "utility scaling" of signals $x$, as well as the coefficient of the risk premium $\sigma_{n}^{2}+V_{j n}^{t}$. To address this, we estimated an alternative version of our baseline model that allows for the coefficient on the risk premium to differ from the coefficient on $x$. We found that the estimated coefficient on the risk premium did not differ appreciably from $\frac{1}{2} \hat{r}^{2}$, where $\hat{r}$ denotes our estimated value for $r$. For that reason, we did not pursue this point further.
} 
type, $k$, that is observed by the both doctor and patient but not by the econometrician. Note also that we only observe patients who obtain at least one prescription (i.e., $w_{0 j}=0$ for all patients $j$ in the sample). Hence, for patient $j$, the likelihood for period $t$ is

$$
\begin{aligned}
& \prod_{n} E_{\epsilon_{j 11}, \ldots, \epsilon_{j 51}}\left(\mathbb{1}\left(W_{j n 1, k}>W_{j n^{\prime} 1, k}, n^{\prime} \neq n\right)\right)^{d_{j n 1}} \quad \text { for } t=1, \\
& E_{\vec{x}_{j n t-1, k}, h_{0 j, k}}\left[\left(\left(1-h_{j t-1, k}\right)\right.\right. \\
& \left.\left.\times \prod_{n} E_{\epsilon_{j 1 t}, \ldots, \epsilon_{j 5 t}}\left(\mathbb{1}\left(W_{j n t, k}>W_{j n^{\prime} t, k}, n^{\prime} \neq n\right)\right)^{d_{j n t}}\right)\right] \\
& \text { for } 1<t<T_{j} \text {, } \\
& E_{\vec{x}_{j n T_{j}, k}, h_{0 j, k}}\left[\left(1-I_{j}\right) * h_{j T_{j}, k}\right] \quad \text { for } t=T_{j}
\end{aligned}
$$

(for the censored observations, we have no information as to whether patient $j$ is healed after period $T_{j}$ or not). If we make the assumption that $\boldsymbol{\epsilon}_{j 1 t}, \ldots, \boldsymbol{\epsilon}_{j 5 t}$ are i.i.d. Type I extreme value, ${ }^{32}$ then the choice probabilities simplify to the multinomial logit expression $E\left(\mathbb{1}\left(W_{j n t, k}>W_{j n^{\prime} t, k}, n^{\prime} \neq n\right)\right)=$ $\exp \left(W_{j n t, k}\right) /\left[\sum_{n^{\prime}=1}^{5} \exp \left(W_{j n^{\prime} t, k}\right)\right] \equiv \lambda_{j n t, k}$. Because a patient's type $k$ is unobserved, the likelihood for all the observations of patient $j$ is

$$
\sum_{k=1}^{K} p_{k} \cdot E_{\vec{x}_{j n T_{j}, k} \mid h_{0 j, k}}\left[\prod_{t=1}^{T_{j}-1}\left(\left(1-h_{j t, k}\right) \prod_{n} \lambda_{j n t, k}^{d_{j n t}}\right)\right] \cdot h_{j T_{j}, k} \prod_{n} \lambda_{j n T_{j}, k}^{d_{j n T_{j}}}
$$

for an uncensored (i.e., $I_{j}=0$ ) observation, and

$$
\sum_{k=1}^{K} p_{k} \cdot E_{\vec{x}_{j n T_{j}, k} \mid h_{0 j, k}}\left[\prod_{t=1}^{T_{j}-1}\left(\left(1-h_{j t, k}\right) \prod_{n} \lambda_{j n t, k}^{d_{j n t}}\right)\right] \cdot \prod_{n} \lambda_{j n T_{j}, k}^{d_{j n T_{j}}}
$$

for a censored (i.e., $I_{j}=1$ ) observation.

\section{Simulation estimation}

In equation (13), the expectations are difficult to calculate because the dimensionality of the joint distribution of $\left(\vec{x}_{j n T_{j}, k}, h_{0 j, k}\right)$ can be large. We therefore use simulation to approximate these expectations. We first draw $S$ vectors

\footnotetext{
${ }^{32}$ This assumption has been made often in the existing literature (cf. Rust (1987), Hotz and Miller (1993)) for computational convenience.
} 
of the unobservables, $\left(k, \vec{x}_{j T_{j}, k}\right)$ for each patient. Then the simulated likelihood for an uncensored patient $j$ is

$$
\frac{1}{S} \sum_{s=1}^{S} \sum_{k=1}^{K} p_{k}\left[\prod_{t}\left(\left(1-h_{j t, k}^{s}\right) \prod_{n}\left(\lambda_{j n t, k}^{s}\right)^{d_{j n t}}\right)\right] \cdot h_{j T_{j}, k}^{s} \prod_{n}\left(\lambda_{j n T_{j}, k}^{s}\right)^{d_{j n T_{j}}}
$$

where $s$ denotes the $s$ th drawn vector of unobservables for patient $j$, and $\lambda_{j n t, k}^{s}$ is the logit choice probability for patient $j$ with type $k$ choosing drug $n$ during period $t$ for the $s$ th draw. (An analogous expression can be derived for the case that patient $j$ 's prescription sequence is censored.) In practice, we use 30 simulation draws per patient when calculating the likelihood function. ${ }^{33}$

\subsection{Identification}

In this section, we briefly consider the structural features of the model and the variation in the data that help to identify the model parameters. The main identification restriction in our bivariate learning model is that a drug's symptomatic effects only impact a patient's utility, while its curative effects only influence the recovery probabilities (cf. (3) and (4)). In this sense, variation in drug choices across patients and prescriptions is important for identifying the parameters related to the symptomatic match value distribution, while variation in recovery frequencies conditional on different sequences of drug choices is important for identifying the parameters related to the curative match value and illness heterogeneity distributions.

Specifically, the parameters related to the symptomatic match value distribution, $\mu_{n}, \underline{\sigma}_{n}^{2}$, and $\sigma_{n}$, as well as the risk aversion parameter $r$, enter the model via the per-period utility expression (3). Market shares at patients' initial prescription help identify the prior symptomatic match values $\mu_{n}$, because doctors are basing their decisions mostly on their prior beliefs at this early juncture of treatment.$^{34}$ Furthermore, because doctors' drug choices late in treatment (especially for those patients with long treatments or those who have tried many drugs) will tend to be based on their true match values, the difference

\footnotetext{
${ }^{33}$ In calculating the standard errors, we use the outer product of the gradient form for the asymptotic approximation of the variance-covariance matrix, which is valid if $S \rightarrow \infty$ and $\sqrt{N} / S \rightarrow 0$ (cf. Gourieroux and Monfort (1996, Chap. 3) and Lee (1992, p. 258)). See Pakes and Pollard (1989) for a general asymptotic theory for estimators obtained by maximizing simulated objective functions and see Lee $(1992,1995)$ for discussions of the asymptotic theory appropriate for the smoothed simulated maximum likelihood procedure employed in this paper.

${ }^{34}$ Unlike static discrete-choice models, however, the $\mu_{n}$ 's are not drug-specific utility intercepts that can freely adjust to reflect overall market shares. The $\mu_{n}$ 's must also adjust to fit dynamic patterns in drug choice.
} 
in the choice probabilities early versus late in treatment will help identify $\underline{\sigma}_{n}^{2}$, which measures the dispersion in symptomatic match values across patients. ${ }^{35}$

The separate identification of the risk-aversion parameter, $r$, and the standard deviations of the signals, $\sigma_{n}$, is more subtle. Risk-averse patients are reluctant to switch drugs, even if their experience to date suggests that their match value for the current drug is low or the drug is expensive. Hence, the degree of risk aversion is identified by the degree of persistence in drug choices. ${ }^{36}$ While the overall incidence of switching identifies $r$, the extent that switching changes with the number of times patients have taken a drug, $l_{j n}^{t}$, identifies $\sigma_{n}$. To see this, note that in the posterior variance formula (7), $\sigma_{n}$ parameterizes how much patients' perceived variances fall-and thus utility and purchase probabilities rise-with increasing experience, as measured by $l$.

Turning to the curative match values and the illness heterogeneity distribution, (10) shows that the quantities $\underline{\nu}_{n}, \underline{\tau}_{n}, \tau_{n}$, and $h_{0 j}$ enter the model only through the expression for the expected recovery probability,

$$
E\left[h_{j t}\left(h_{j t-1}, y_{j n t}\right) \mid \mathcal{S}_{t}\right]=E_{\nu_{j n}} E_{y_{j n t} \mid \nu_{j n}}\left[\frac{\left(\frac{h_{j t-1}}{1-h_{j t-1}}\right)+d_{j n t} y_{j n t}}{1+\left(\left(\frac{h_{j t-1}}{1-h_{n t-1}}\right)+d_{j n t} y_{j n t}\right)} \mid \mathcal{S}_{t}\right],
$$

where the first expectation is taken over $N\left(\nu_{j n}^{t}, R_{j n}^{t}\right)$, the period- $t$ posterior distribution of $\nu_{j n} \mid \mathcal{S}_{t}$. Since the parameters $\underline{\nu}_{n}, \underline{\tau}_{n}$, and $\tau_{n}$ enter only in the expressions for the posterior mean $\nu_{j n}^{t}$ and variance $R_{j n}^{t}$ (cf. (8)), and the illness severity $h_{0 j}$ enters only in the expression for the healing probabilities, $h_{j t-1}$ (cf. (4)), these parameters are jointly identified.

The particular exponential functional form chosen for the per-period utility function (3) is important in separately identifying the mean symptomatic match values, $\mu_{n}$, from the price parameter, $\alpha$. Because neither $\mu_{n}$ nor the drug prices vary over time, we would not be able to separately identify $\mu_{n}$ and $\alpha$ in a utility specification in which the posterior symptomatic mean $\mu_{j n}^{t}$ and the drug price enter linearly, since both would be collapsed into a drug-specific intercept term. In our exponential specification, however, $\mu_{j n}^{t}$ enters interacted with $V_{j n}^{t}$,

\footnotetext{
${ }^{35}$ Specifically, as $\underline{\sigma}_{n} \rightarrow 0$, all patients will have the same match values, identically equal to the prior match values $\underline{\mu}_{n}, n=1, \ldots, 5$. This implies that market shares near the end of treatment should equal market shares at the beginning of treatment.

${ }^{36}$ As noted by a referee, persistence in drug choices can be generated not only by risk aversion, but also by serial correlation in perceived qualities, $\mu_{j n}^{t}$, induced by patients' match values. The rational expectations assumption is important in overcoming this identification problem, because it implies that high and low signals (relative to the prior mean match value) are equally likely after a patient's first use of a drug. Hence, a patient is equally likely to "update up" as "update down" from her first use of this drug so that, without risk aversion, the repeat purchase probability after this first use should be, on average, no higher than her purchase probability before this use. In contrast, risk aversion always generates persistence after the first use via reductions in the risk premium, so that the repeat purchase probability after first use should be, on average, much higher than her purchase probability before this use. This is what we find in the data.
} 
the (time-varying) perceived variance. Hence, $\mu_{n}$ is identified from variation in drug choices across patients and prescriptions given different levels of experience, leaving cross-sectional variation in market shares to identify the price coefficient, $\alpha .^{37}$ Finally, unobserved heterogeneity in initial illness severity appears necessary to convincingly explain some features of the data. ${ }^{38}$

\section{ESTIMATION RESULTS}

Tables III and IV contain the estimates for the parameters of our bivariate dynamic learning model. ${ }^{39}$ The first panel of Table III presents our estimates of the patient type distribution. As suggested by (2), we allow for a discrete distribution of patient types. ${ }^{40}$ We began by allowing for two unobserved types $(K=2)$ and then increased the number until there were negligible changes in both model fit and the qualitative conclusions from the model. This yielded an estimated four type parameters.

As described earlier, each patient type begins treatment with a different initial illness severity and draws its drug-specific match values from different distributions. Hence, for each of the four patient types, we estimate different initial recovery probabilities and mean match values $\underline{\mu}_{1}, \ldots, \underline{\mu}_{5}, \underline{\nu}_{1}, \ldots, \underline{\nu}_{5}$.

\section{Discussion of results}

The parameter estimates for the distribution of patient types provides strong support for illness heterogeneity in the population of ulcer sufferers. The first panel of Table III reports that Type 1 patients are those with mild conditions who begin with a $43.3 \%$ chance of recovering after their first prescription and make up $59.3 \%$ of the sample. Type 2 patients are the sickest patients, who

\footnotetext{
${ }^{37}$ While the specific functional forms for the utility function are important, we performed simulations of the model to ensure that certain empirical implications of this utility specification are confirmed in the actual data. The positive results from these simulations support our specification. We thank a referee for this suggestion.

${ }^{38}$ Without illness severity, for example, the large number of patients who are healed after a single prescription to the composite drug 5 yields - contrary to the medical evidence-a high estimated curative value for that drug. Once we allow for unobserved illness heterogeneity, however, the curative match values estimated for drug 5 decrease substantially because such short treatments may now be attributed to mild conditions (permitting the curative match value to be identified by the (in)effectiveness of drug 5 at treating longer sequences).

${ }^{39}$ In our empirical specification, we imposed several additional restrictions on the parameters. First, while we allow the variances of the symptomatic signals, $\sigma_{n}^{2}$, to vary across drugs, we restrict the variance of the curative signals to be identical across drugs $\left(\tau_{n}^{2} \equiv \tau^{2}, \forall n\right)$. Furthermore, the estimated prior variances, $\hat{\sigma}_{n}^{2}$, were quite similar, so we imposed the restriction that they are identical across drugs, $\underline{\sigma}_{n}^{2} \equiv \underline{\sigma}^{2}, \underline{\tau}_{n}^{2} \equiv \underline{\tau}^{2}, \forall n$.

${ }^{40}$ This follows Heckman and Singer (1984). While the results are not reported here, we have also tried continuous heterogeneity distributions, including Gamma and a mixture of Normals. The estimates for these specifications supported an extremely skewed distribution that seemed to us well approximated by the four-point discrete distribution given in (2).
} 
TABLE III

DYNAMIC MODEL: PARAMETER ESTIMATES

\begin{tabular}{|c|c|c|c|c|}
\hline Parameter & Est. & Std. Err. & Est. & Std. Err. \\
\hline Illness heterogeneity distribution & \multicolumn{2}{|c|}{ Recovery Probability } & \multicolumn{2}{|c|}{ Type Probability } \\
\hline$\theta_{1}$ (Type 1 ) & 0.433 & 0.003 & 0.593 & 0.006 \\
\hline$\theta_{2}$ (Type 2 ) & 0.127 & 0.003 & 0.335 & 0.006 \\
\hline$\theta_{3}$ (Type 3 ) & 0.199 & 0.007 & 0.043 & 0.001 \\
\hline$\theta_{4}$ (Type 4) & 0.432 & 0.011 & 0.029 & 0.002 \\
\hline Means, symptom match values ${ }^{\mathrm{b}}$ & \multicolumn{2}{|c|}{ Type 1} & \multicolumn{2}{|c|}{ Type 2} \\
\hline$\mu_{1}$ & 0.927 & 0.282 & 1.195 & 0.369 \\
\hline $\bar{\mu}_{2}{ }^{\mathrm{c}}$ & 0.928 & 0.287 & 0.428 & 0.166 \\
\hline $\bar{\mu}_{3}$ & 0.481 & 0.197 & -0.028 & 0.178 \\
\hline $\bar{\mu}_{4}$ & 0.335 & 0.161 & -0.145 & 0.079 \\
\hline $\bar{\mu}_{5}$ & 0.451 & 0.174 & -0.483 & 0.137 \\
\hline$\overline{\text { Means, curative match values }}{ }^{\mathrm{b}}$ & \multicolumn{2}{|c|}{ Type 1} & \multicolumn{2}{|c|}{ Type 2} \\
\hline$\underline{\nu}_{1}$ & 0.014 & 0.003 & 0.006 & 0.000 \\
\hline$\underline{\nu}_{2}^{\mathrm{c}}$ & 0.015 & 0.005 & 0.006 & 0.001 \\
\hline$\underline{\nu}_{3}$ & 0.013 & 0.030 & 0.006 & 0.095 \\
\hline$\underline{\nu}_{4}^{3}$ & 0.013 & 0.084 & 0.014 & 0.009 \\
\hline $\begin{array}{l}-4 \\
\nu_{5}\end{array}$ & -0.034 & 0.000 & -0.038 & 0.000 \\
\hline $\begin{array}{l}\text { Std. dev., symptom match values } \\
\underline{\sigma}\end{array}$ & 1.574 & 0.448 & & \\
\hline \multicolumn{5}{|l|}{$\overline{\text { Std. devs., symptom signals }}$} \\
\hline$\sigma_{1}$ & 0.998 & 0.287 & & \\
\hline$\sigma_{2}$ & 1.134 & 0.326 & & \\
\hline$\sigma_{3}$ & 1.375 & 0.395 & & \\
\hline$\sigma_{4}$ & 1.159 & 0.333 & & \\
\hline$\sigma_{5}$ & 0.931 & 0.268 & & \\
\hline \multicolumn{5}{|l|}{ Std. dev., curative match values } \\
\hline$\underline{\tau}$ & 0.007 & 0.000 & & \\
\hline \multicolumn{5}{|l|}{$\overline{\text { Std. dev., curative signals }}$} \\
\hline$\tau$ & 0.007 & 0.001 & & \\
\hline Price coefficient, $\alpha^{\mathrm{a}}$ & 1.080 & 0.091 & & \\
\hline Risk-aversion parameter, $r$ & 0.990 & 0.274 & & \\
\hline Discount rate, $\beta$ & 0.950 & Fixed & & \\
\hline Number of observations & 34,972 & & & \\
\hline Number of similar draws & 30 & & & \\
\hline Log likelihood function & $-124,484.34$ & & & \\
\hline
\end{tabular}

\footnotetext{
${ }^{\text {a }}$ Price in thousands of lire.

${ }^{b}$ Prior symptomatic and curative means are reported for two highest probability types. For prior means corresponding to all types, see Table IV.

${ }^{\mathrm{c}}$ Prior means for drug 2 are reported for the Jul-Dec 1992 period. For prior means corresponding to earlier periods, see Table IV.
} 
TABLE IV

Dynamic Model: PARAMETER Estimates (CONT'D): MATCH VAlues FOR All TyPeS AND ACROSS TIME FOR OMEPRAZOLE

\begin{tabular}{|c|c|c|c|c|c|c|c|c|}
\hline \multirow[b]{2}{*}{ Parameter } & \multicolumn{2}{|c|}{ Type 1} & \multicolumn{2}{|c|}{ Type 2} & \multicolumn{2}{|c|}{ Type 3} & \multicolumn{2}{|c|}{ Type 4} \\
\hline & Est. & Std. Err. & Est. & Std. Err. & Est. & Std. Err. & Est. & Std. Err. \\
\hline \multicolumn{9}{|c|}{ Match values, all types } \\
\hline \multicolumn{9}{|c|}{ Symptom match values } \\
\hline$\underline{\mu_{1}}$ & 0.927 & 0.282 & 1.195 & 0.369 & 0.489 & 0.163 & 0.151 & 0.091 \\
\hline$\overline{\bar{\mu}}_{2}{ }^{\mathrm{a}}$ & 0.928 & 0.287 & 0.428 & 0.166 & 0.577 & 0.198 & 0.573 & 0.199 \\
\hline $\bar{\mu}_{3}$ & 0.481 & 0.197 & -0.028 & 0.178 & 1.762 & 0.531 & 0.013 & 0.167 \\
\hline$\overline{\bar{\mu}}_{4}$ & 0.335 & 0.161 & -0.145 & 0.079 & -0.111 & 0.305 & 0.504 & 0.184 \\
\hline$\overline{\bar{\mu}}_{5}$ & 0.451 & 0.174 & -0.483 & 0.137 & -0.113 & 0.125 & -0.561 & 0.220 \\
\hline \multicolumn{9}{|c|}{ Curative match values } \\
\hline$\underline{\nu}_{1}$ & 0.014 & 0.003 & 0.006 & 0.000 & 0.011 & 0.002 & 0.014 & 0.010 \\
\hline$\underline{\nu}_{2}^{\mathrm{a}}$ & 0.015 & 0.005 & 0.006 & 0.001 & 0.011 & 0.006 & 0.015 & 0.003 \\
\hline$\underline{\nu}_{3}$ & 0.013 & 0.030 & 0.006 & 0.095 & 0.004 & 0.001 & 0.013 & 0.329 \\
\hline$\underline{\nu}_{4}$ & 0.013 & 0.084 & 0.014 & 0.009 & -0.035 & 0.214 & 0.012 & 0.003 \\
\hline$\underline{\nu}_{5}$ & -0.034 & 0.000 & -0.038 & 0.000 & -0.037 & 0.054 & -0.034 & 0.409 \\
\hline
\end{tabular}

Time-varying priors for omeprazole

Symptom match value, $\underline{\mu}_{2}$

\begin{tabular}{lrrrrrrrr} 
Period 1 & 0.805 & 0.258 & 0.306 & 0.140 & 0.454 & 0.171 & 0.451 & 0.172 \\
Period 2 & 0.910 & 0.285 & 0.411 & 0.166 & 0.560 & 0.197 & 0.556 & 0.198 \\
Period 3 & 0.722 & 0.237 & 0.223 & 0.122 & 0.371 & 0.151 & 0.368 & 0.152 \\
Period 4 & 0.979 & 0.301 & 0.480 & 0.181 & 0.628 & 0.212 & 0.625 & 0.214 \\
Period 5 $^{\mathrm{a}}$ & 0.928 & 0.287 & 0.428 & 0.166 & 0.577 & 0.198 & 0.573 & 0.199 \\
Curative match value, $\underline{\nu}_{2}$ & & & & & & & \\
Period 1 & -0.007 & 0.011 & -0.016 & 0.010 & -0.011 & 0.011 & -0.007 & 0.010 \\
Period 2 & -0.001 & 0.012 & -0.011 & 0.011 & -0.006 & 0.012 & -0.001 & 0.011 \\
Period 3 & 0.015 & 0.016 & 0.005 & 0.015 & 0.011 & 0.016 & 0.015 & 0.016 \\
Period 4 $^{\text {Period 5 }}$ & 0.013 & 0.017 & 0.004 & 0.016 & 0.009 & 0.017 & 0.013 & 0.017 \\
& 0.015 & 0.005 & 0.015 & 0.001 & 0.011 & 0.006 & 0.015 & 0.003 \\
\hline
\end{tabular}

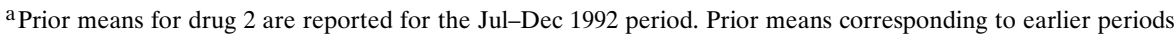
are reported in the bottom panel of this table.
}

begin treatment with only a $12.7 \%$ chance of recovering and make up $33.5 \%$ of the sample. The remaining $8.2 \%$ of patients are Type 3 and Type 4 patients, who begin treatment with a $19.9 \%$ and $43.2 \%$ initial recovery probability.

Table III reports the parameters of the match value distributions for the Type 1 ("not-so-sick") and Type 2 ("sick") patients that make up over $90 \%$ of the sample. ${ }^{41}$ The point estimates for the parameters of the symptomatic match value distribution indicate substantial heterogeneity in match values across patients. First, the estimates of the mean match values $\underline{\mu}_{1}, \ldots, \underline{\mu}_{5}$ indicate that the ranking of these drugs along the symptom-relief dimension is $(2,1,3,5,4)$

${ }^{41}$ Table IV presents the same estimates for all the patient types. 
for Type 1 patients and $(1,2,3,4,5)$ for Type 2 patients. The estimate of the standard deviation of symptomatic match values, $\underline{\sigma}$ (1.58), is large when compared to the magnitudes of the across-drug differences in the mean match values. In Figure 2, we graph the estimated densities for the symptomatic match values for these two patient types. The substantial overlap in the supports of these density functions exhibited in each graph implies that, for all patient types, the drugs are clearly horizontally differentiated, so that the symptomatic ranking among the drugs is very likely to be different even for two patients of the same patient type. Since match values are unknown to patients and doctors at the beginning of treatment, the heterogeneity in match values implied by these results highlights the importance of the matching problem in this market.

The point estimates obtained for the parameters of the curative match value distribution provide further evidence of heterogeneity in match values across patients. The average ranking of drugs in this dimension is $(2,1,3,4,5)$ for the Type 1 patients and $(4,1,2,3,5)$ for the Type 2 patients. Significantly, the composite drug 5 is estimated to have negative curative mean match values on average for all patient types. Moreover, the estimated magnitude of $\underline{\tau}$ is large compared to differentials in the mean curative match values $\underline{\nu}_{n}$ across drugs, which indicates substantial horizontal differentiation across patients along the curative dimension, just as with the symptomatic match value densities plotted in Figure 2.

In Table IV we present the symptomatic and curative match values for all four patient types as well as the trend in the estimated prior match value means for omeprazole. The top half of Table IV reports that Type 3 patients, representing $4.3 \%$ of the sample, are fairly ill and prefer the symptomatic effects of drug 3, while Type 4 patients, representing $2.9 \%$ of the sample, prefer drugs 2 and 4 . Whereas Types 3 and 4 represent a small share of the patient population, we do not focus further on these results.

The bottom half of Table IV presents the estimated evolution in doctors' prior beliefs for omeprazole, the innovative molecule that entered at the beginning of our sample. We see that, as might be expected, these prior means generally rise over time-particularly in the curative dimension-implying that beliefs about the efficacy of omeprazole became more optimistic over time. This indicates that, for omeprazole, there are important sources of learning by doctors that are driving prescription behavior. This is not surprising, given that omeprazole is the newest drug in the sample and that its active ingredient is markedly different from the active ingredients of the other available drugs.

A number of our results reconcile important differences between inferences of drug quality obtained from market shares versus the medical literature in the anti-ulcer market. As might be expected of a market leader, drug 1 (ranitidine) performs well in all dimensions among all types of patients. Drug 2, (omeprazole), is a therapeutic innovation that, despite having a significantly lower market share (and a higher price) than drug 1 in the data, is nonetheless estimated 
Type 1 Patients

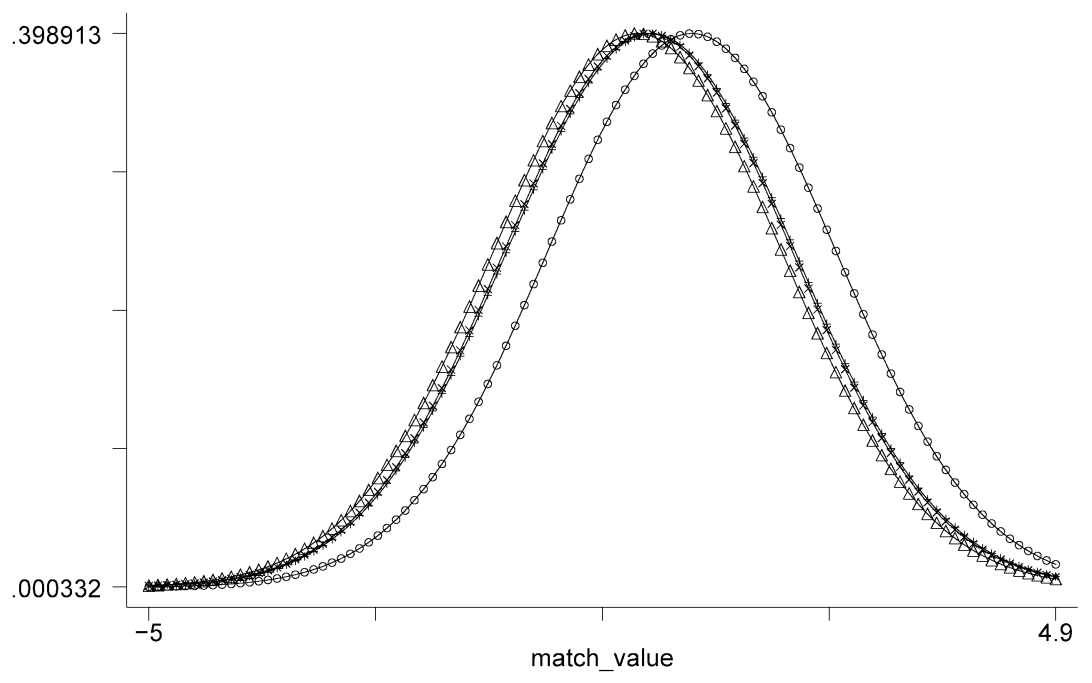

Type 2 Patients

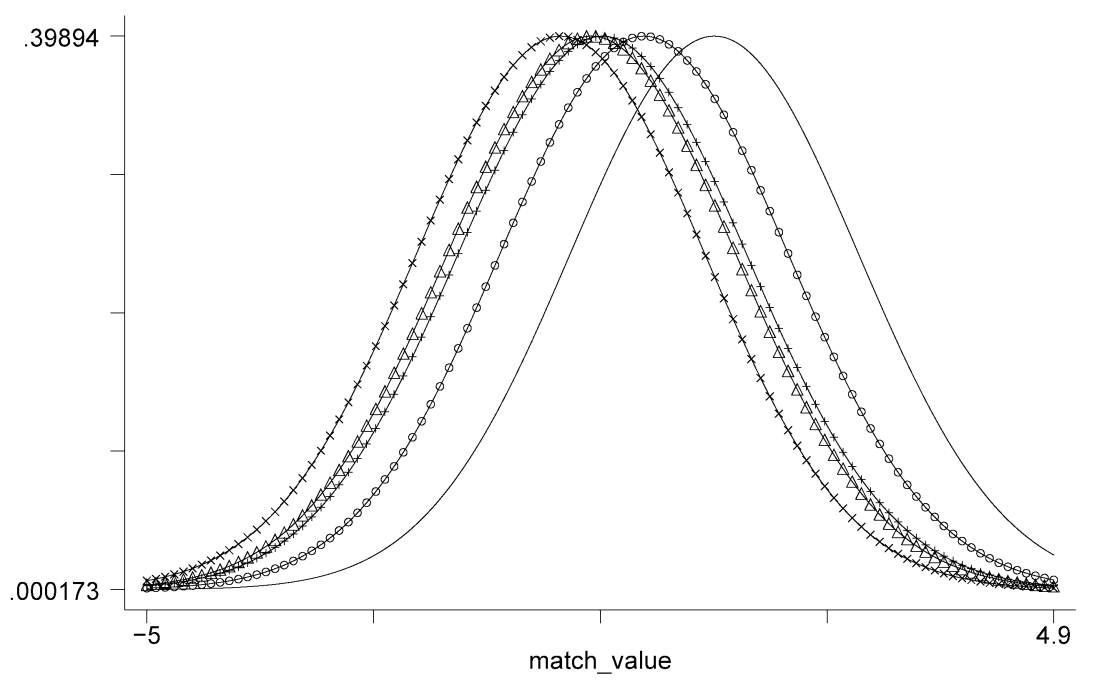

FIGURE 2.-Heterogeneity in symptomatic match values: Type 1 and Type 2 patients. Density of symptomatic match values $\mu_{j n}$ across patients. Solid line, drug 1 (ranitidine); circle, drug 2 (omeprazole); plus, drug 3 (famotidine); triangle, drug 4 (nizatidine); cross, drug 5 (small brands).

to have a slightly higher mean match value to drug 1 in the curative dimension for Type 1 patients and comparable curative match values for the other patient types. Furthermore, drug 2's relatively poor symptomatic performance among 
sicker Type 2 patients (its mean symptomatic match value of 0.428 is a distant second behind drug 1's value of 1.195) is consistent with medical evidence about its approval for use..$^{42}$ On the other hand, drug 5, a composite of older, milder drugs, appears effective only in relieving symptoms for the least sick Type 1 patients, who presumably also have milder symptoms than the other patient types. Finally, our estimates of the standard deviations of the symptomatic signals, $\sigma_{1}, \ldots, \sigma_{5}$, indicate that drugs 1 and 5 yield less noisy signals than drugs $2-4$, which is consistent with the observations that the former drugs are older than the latter drugs and that the experience signals may be expected to be more precise for the older drugs.

\subsection{Model Fit}

Before considering some broader implications of our results, we consider the fit of our estimated model. To do so, we generate simulated prescription sequences using our estimated parameters and compare summary statistics from this data with the analogous statistics for the actual estimating data. While the observed prescription sequences are censored, the simulated sequences are not. Therefore, to better assess model fit, we censor the simulated data in a manner consistent with the censoring in the data set and compare the market shares, treatment costs, and treatment lengths after doing this "simulated censoring." ${ }^{43}$

Table V contains average treatment lengths, treatment costs, and market shares calculated from the actual data as well as from a simulated data set of 5,000 hypothetical patients. ${ }^{44}$ Turning to the first column of Table V, we see that both the average treatment length and the treatment cost match remarkably

\footnotetext{
${ }^{42}$ In particular, upon initial introduction, drug 2 (omeprazole) was not approved for "maintenance therapy" or long-term treatment of some chronic or persistent conditions (cf. Yamada (1995, pp. 1233-1234)).

${ }^{43}$ We begin by constructing a sampling distribution of censoring times that is the empirical distribution of the censoring times observed in the data. In constructing this distribution, we follow the assumptions on right censoring presented in Section 4. For the patients with censored prescription sequences (those for whom we observe prescriptions during months 31-36 (i.e., July-Dec 1992) of the sample), we take the observed treatment length as the censoring time. For the patients with uncensored prescription sequences (those for whom we do not observe prescriptions during months $31-36$ of the sample), the censoring time is unobserved. For these patients, we construct the censoring time as $31-\pi_{0}$, where $\pi_{0}$ is the first month in which we observe a prescription for this patients; $\pi_{0}$ is measured as the number of months after January 1990. After constructing this sampling distribution of censoring times, we draw a censoring time for each simulated patient from this distribution and truncate each simulated prescription sequence at this censoring time if the length of the simulated prescription sequence exceeds the censoring time.

${ }^{44}$ We also considered the out-of-sample fit of our model using data from Jan-Jun 1990, which were not used in the estimation. The results of this exercise were confounded by left censoring in the data, however, because the patients in this data likely commenced treatment before the sample period began. As such, we do not report the results here.
} 
TABLE V

Model Fit: MARKET SHARES AND TREATMENT CHARACTERISTICS

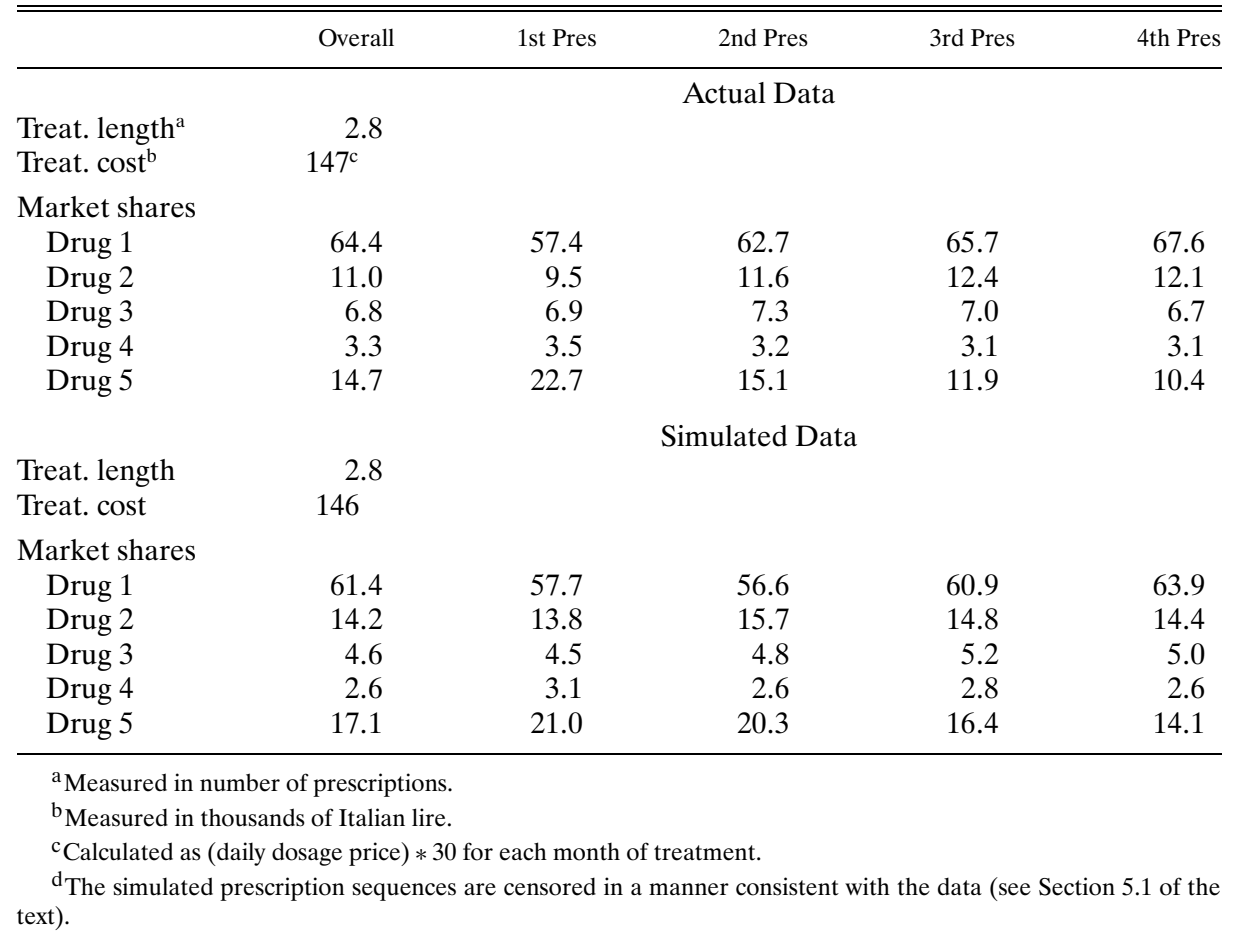

well in the simulated data. Furthermore, the simulated overall market shares match the actual market shares in ranking, but the market share of drug 1 , ranitidine, is slightly underpredicted, while the share of drug 5 is overpredicted.

The remaining columns of Table $\mathrm{V}$ consider how well our specifications match dynamic features of the data. The top panel of Table $\mathrm{V}$ presents market shares in the data at the first through fourth prescriptions across all patients. Two trends are apparent: the market share for drug 1 rises over prescriptions and that for drug 5 falls over prescriptions. The bottom panel of Table V presents the analogous shares calculated using the simulated data. While the magnitudes deviate somewhat from the actual data, both the upward trend in market share for drug 1 and the downward trend in drug 5 detected in the actual data are duplicated in the simulated data.

Given our model's close fit of the average treatment length, we also consider how well our specifications fit observed treatment lengths and recovery probabilities. In Figure 3, we present plots of the empirical survival functions for both the actual and simulated data. The fit here is also very encouraging: for example, the survival function in the actual data at three and four prescriptions is roughly $21 \%$ and $15 \%$, respectively, while the corresponding percentages in 


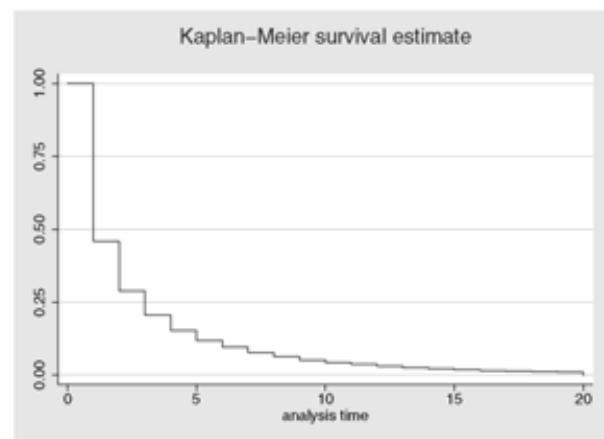

Actual Data

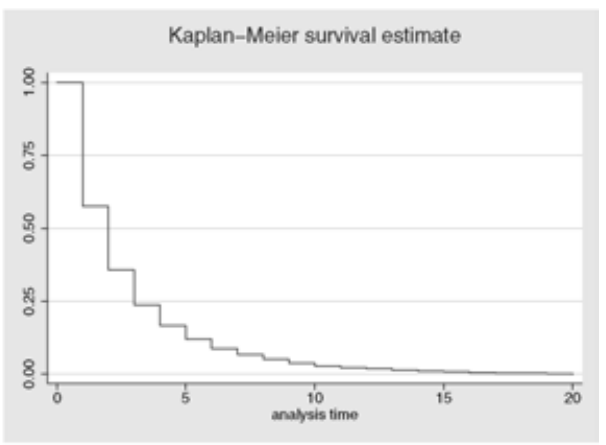

Simulated DAta

FIGURE 3.-Empirical survival estimates. All sequences truncated at 20 prescriptions to provide ease of comparison.

the simulated data are just very close at $24 \%$ and $17 \%$. The only deviation between observed and simulated data is a slight overprediction of recovery probabilities for short treatments and corresponding underprediction of recovery for long treatments.

\section{UNCERTAINTY, EXPERIMENTATION, AND ITS CONSEQUENCES}

We next use our estimates to assess the importance of uncertainty, learning, and experimentation in generating the prescription sequences observed in the data. We begin by describing the implications of our estimates for the speed at which patients and their doctors learn whether a drug is effective for the patient. Our estimates imply that learning occurs quite quickly. For example, for drug 1, a Type 1 patient's perceived variance for symptomatic relief falls from 2.48 to 0.70 after a single prescription, a decrease of over $70 \%$. The incremental effects of subsequent prescriptions are fairly low-the majority of uncertainty reduction (and consequent increase in utility to the first-chosen drug) comes with first use. Similar results obtain for the speed at which patients learn about drugs' curative effects. The very large effects of the first prescription implies significant switching costs to patients in pharmaceutical markets. ${ }^{45}$

\footnotetext{
${ }^{45}$ While our focus is on the matching process between patients and drugs based on experience, a referee has noted that it is critical that we accommodate as well as possible the "diagnostic matching" of patients to drugs by doctors prior to drug use. Failing to do so will tend to attribute persistence in drug choices to reduced uncertainty and risk aversion rather than the accurate diagnosis of a particular drug for a particular condition. We have explored the sensitivity of our results to alternative specifications of the distribution of patient types (equivalently, diagnosis types) and found them to be very robust. Later in this section, we present results that measure the relative importance of matching based on doctors' diagnoses versus patients' experience that finds both effects to be empirically important.
} 
Our estimates of $r$, the risk aversion parameter (0.990), and the price coefficient $\alpha(1.080)$ indicate that a Type 1 patient would value this decrease in the perceived variance at about 950 lire, which is over $65 \%$ of her copayment per daily dose of drug 1 .

While patients appear to learn quickly, there is still the question of the costs, both monetary and otherwise, of uncertainty in anti-ulcer markets. To assess these costs, we simulate prescription sequences for 5,000 hypothetical patients under two counterfactual assumptions. In the first counterfactual, we examine drug choice in the "best-case" scenario where patients have complete information about their match values. To do so, we continue to have patients draw symptomatic and curative match values from the population distributions, $N\left(\underline{\mu}_{n k}, \underline{\sigma}_{n k}\right)$ and $N\left(\underline{\nu}_{n k}, \underline{\tau}_{n k}\right)$, but set their perceived variance, $V_{j n}^{t}$ and $R_{j n}^{t}$, to zero. ${ }^{46}$ In the second counterfactual, we preclude experimentation by constraining patients to continue taking the first drug they are prescribed, thus effectively shutting down learning after the first prescription. By comparing the results from these simulations to the baseline values implied by our estimates, we isolate the effects of uncertainty and experimentation in this market and assess how actual choices compare to those made when each is removed.

The first panel of Table VI presents baseline estimates of average patient utility, treatment length, treatment costs, and market shares for each drug implied by the model at the estimated parameter values. Note that the reported results here are for the uncensored simulated treatment sequences, which differ slightly from the results presented in Table V for the censored simulated treatment sequences.

The second panel of Table VI summarizes patient choices under complete information. The average number of drugs taken during treatment increases by a third and market shares become much less concentrated, leading to a sharp fall in the Herfindahl Index (from 4,242 to 2,676). As expected, discounted expected utility increases. ${ }^{47}$ The implications of these counterfactuals are quite interesting. With complete information, one might expect patients to simply purchase the drug that yields their highest match and remain with it. Instead, the average number of drugs increases (from 1.4 to 1.9). This occurs as patients optimize their choice of drugs over the course of treatment. The increase in market share of all the drugs at the expense of the market leader is unsurprising. As suggested in Figure 2, our estimates suggest drugs' symptomatic match values do not differ greatly, but that there are switching costs induced by patient-level uncertainty that favor the first-chosen drug.

\footnotetext{
${ }^{46}$ Note that, even with complete information, patients under this counterfactual still face uncertainty about the symptomatic and curative signals they receive after each prescription. However, by removing all uncertainty about their match values, all incentives to experiment are removed.

${ }^{47}$ While it appears that average treatment length and treatment cost increase considerably, this is an artifact of a small number of very long simulated treatment sequences: indeed, the median treatment cost actually is smaller under this counterfactual than in the baseline specification.
} 
TABLE VI

RESULTS FROM COUNTERFACTUAL SIMULATIONS

\begin{tabular}{|c|c|}
\hline \multicolumn{2}{|c|}{ Baseline Specification $^{\mathrm{a}}$} \\
\hline Avg. discounted utility & -28.7 \\
\hline Avg. treatment length & 4.8 \\
\hline Avg. treatment cost & 245 \\
\hline Avg. number of different drugs & 1.4 \\
\hline \multicolumn{2}{|l|}{ Market shares } \\
\hline Drug 1 & 60.4 \\
\hline Drug 2 & 14.1 \\
\hline Drug 3 & 3.7 \\
\hline Drug 4 & 2.5 \\
\hline Drug 5 & 19.3 \\
\hline Herfindahl index & 4,242 \\
\hline \multicolumn{2}{|c|}{ Counterfactual I: Complete Information ${ }^{\text {b }}$} \\
\hline Avg. discounted utility & -26.4 \\
\hline Avg. treatment length & 8.8 \\
\hline Avg. treatment cost & 385 \\
\hline Avg. number of different drugs & 1.9 \\
\hline \multicolumn{2}{|l|}{ Market shares } \\
\hline Drug 1 & 22.4 \\
\hline Drug 2 & 12.9 \\
\hline Drug 3 & 12.0 \\
\hline Drug 4 & 10.9 \\
\hline Drug 5 & 41.8 \\
\hline Herfindahl index & 2,676 \\
\hline \multicolumn{2}{|c|}{ Counterfactual II: No Experimentation ${ }^{\mathrm{c}}$} \\
\hline Avg. discounted utility & -30.6 \\
\hline Avg. treatment length & 4.8 \\
\hline Avg. treatment cost & 248 \\
\hline
\end{tabular}

\footnotetext{
${ }^{\text {a }}$ These figures differ from those given in the bottom panel of Table $\mathrm{V}$ because they were computed using uncensored simulated prescription sequences, while those reported in Table V were calculated using censored simulated prescription sequences.

${ }^{\mathrm{b}}$ In these simulations, we assume that each patient $j$ knows her match values $\mu_{j}$ and $\nu_{j}$. Therefore, while signals are still stochastic, patients' perceived variances, $V_{j n}^{r}$ and $R_{j n}^{t}$, are set equal to zero.

${ }^{\mathrm{c}}$ In these simulations, we restrict patients to take their first drug for the duration of their entire treatment.
}

Furthermore, we note that removing uncertainty does not yield a dramatic change in average discounted utilities. In the complete information case, the average discounted utility is -26.4 , about $9 \%$ higher than the average discounted utility obtained in the baseline case with uncertainty. This reduction in utility levels is smaller than the reduction that occurs when experimentation is prohibited, as illustrated in the second counterfactual, reported in the bot- 
tom panel of Table IV. Requiring patients to continue taking their first drug for the entire duration of their treatment does not change simulated treatment lengths and moderately increases simulated treatment costs, but lowers the average utility level down to -30.1 , which is about $6 \%$ below the baseline case, and which allows for learning and experimentation. ${ }^{48}$ The decrease in utility levels when experimentation is precluded highlights the role of learning and experimentation in enabling patients to realize discounted utility levels that are closer to those attainable in a first-best scenario with complete information.

\section{On diagnostic versus experiential matching}

As described earlier, doctors in our model perform the "diagnostic matching" of patients to drugs prior to writing any prescriptions. How important is this diagnostic matching when compared to matching based on patients' experience with the alternative drugs? Table VII evaluates this issue by calculating the discounted expected utility, treatment lengths, treatment costs, and market shares for a counterfactual experiment that eliminates diagnostic matching by doctors. In this counterfactual, we assume that doctors are unable to uncover patient types during the initial diagnosis. To impose this restriction, we set the prior mean (in both the symptomatic and the curative dimensions) for each drug to be the weighted average of the type-specific prior means, with the weights given by the estimated type probabilities $p_{1}, \ldots, p_{4}$.

TABLE VII

RESULTS FROM COUNTERFACTUAL SIMULATIONS (CONT’D)

\begin{tabular}{lc}
\hline \hline \multicolumn{2}{c}{ Counterfactual III: No Diagnostic Matching ${ }^{\mathrm{a}}$} \\
\hline Avg. discounted utility & -31.9 \\
Avg. treatment length & 6.9 \\
Avg. treatment cost & 324 \\
Avg. number of different drugs & 1.6 \\
Market shares & \\
Drug 1 & 41.6 \\
Drug 2 & 18.3 \\
Drug 3 & 2.2 \\
Drug 4 & 2.4 \\
Drug 5 & 35.4 \\
Herfindahl index & 3,333 \\
\hline
\end{tabular}

\footnotetext{
${ }^{\mathrm{a}}$ In these simulations, we assume that doctors are unable to determine the initial illness severity $h_{0 j}$ for each patient during the initial diagnosis. To impose this restriction, we set the prior mean (in both the symptomatic and curative dimensions) for each drug to be the weighted average of the type-specific prior means, with the weights given by the estimated type probabilities $p_{1}, \ldots, p_{4}$.
}

${ }^{48}$ We thank a referee and Wilbert van der Klaauw for suggesting this counterfactual. 
Table VII shows that expected utility is approximately $11.1 \%$ lower, and expected treatment costs and length are 30-40\% higher than in the baseline scenario with diagnostic matching. Indeed the relative loss in utility/increase in treatment costs and length from diagnostic matching is actually greater than the comparable gain that would obtain under complete information presented in the second panel of Table VI. This suggests that the diagnostic process is at least as important to patients as the idiosyncratic learning that occurs as patients match to their preferred drug. Interestingly, the "pooling of types" caused by eliminating doctor diagnoses yields a less concentrated market, suggesting that while diagnostic matching of patients to drugs clearly benefits patients, it does so at the expense of greater market power for leading drugs.

\section{CONCLUDING REMARKS}

In this paper, we measure the importance of uncertainty and learning in the demand for pharmaceutical drugs. To do so, we specify a dynamic matching model of demand in which doctors choose a sequence of drugs to minimize their patients' expected disutility from illness and learn over time about the effectiveness of available drugs for their particular condition. We extend existing models of dynamic demand in two ways. First, we allow drugs to be differentiated in two dimensions, having both symptomatic and curative effects. Second, we endogenize patients' length of treatment by allowing their drug choices to impact their probability of recovery.

Our results indicate that these extensions are empirically important in the market for anti-ulcer drugs. Drugs' symptomatic and curative rankings differ in ways that are consistent with the medical literature. We also find strong evidence of learning: despite substantial initial uncertainty about drugs' effectiveness (in each dimension), there are sharp reductions in this uncertainty after even a single prescription. We also find that patients are risk-averse, implying a strong disincentive to switch away from their first-chosen drug. Despite significant uncertainty in this market, counterfactual simulations demonstrate that learning and experimentation enable patients to realize discounted utility levels that are quite close to those attainable in a first-best scenario with complete information.

The estimated differences in drugs' symptomatic and curative effects suggests incorporating outcome measures (as here, a patient's length of treatment) may be an important feature to include in the structural analysis of consumer demand. The extension to medical markets beyond pharmaceuticals is clear, but similar approaches may be appropriate for markets with an important endogenous temporal dimension, such as markets for "experts" like legal or business services where performance can be directly (if imperfectly) measured ex post. More broadly, our evidence on uncertainty and risk aversion suggests substantial switching costs in pharmaceutical markets, even after a single prescription. Inducing strong prior beliefs about drug quality via (especially 
direct-to-consumer) promotional expenditures can therefore dramatically increase profitability and market concentration, even when alternative drugs are therapeutically similar. This suggests that designing policies to improve information diffusion in drug markets might play an important competitive role. We plan to examine these issues in future work.

Department of Economics, Eller College of Management, University of Arizona, 401 McClelland Hall, Tucson, AZ 85721-0108, U.S.A.; crawford@eller.arizona. edu; http://ellerarizona.edu/ crawford

\section{and}

Department of Economics, Johns Hopkins University, 3400 North Charles Street, Baltimore, MD 21218, U.S.A.; mshum@jhu.edu; http://www.econ.jhu.edu/ people/shum.

Manuscript received March, 2000; final revision received January, 2005.

\section{APPENDIX: VALUE FUNCTION APPROXIMATION ALGORITHM}

We adapt the Keane and Wolpin (1994) approximation method for discretechoice dynamic programming (DCDP) problems to our model. In essence, the value function is evaluated at only a subset of the (perhaps very large number of) points in the state space, and a specific form of interpolation is used to approximate the value function. Recall the general setting (for the sake of generality, the notation here is simplified from that given in the main text):

- A patient solves the infinite-horizon optimization problem

$$
\max _{\left\{D_{t}\right\}_{t=1}^{\infty}} E \sum_{t=1}^{\infty} \beta^{t-1} \sum_{k=1}^{K} u_{k}(S(t)) D_{t},
$$

where, each period, one of $K$ discrete choices must be taken (and where $D_{t}$ is a $K$ vector whose $k$ th element equals 1 if choice $k$ is taken during period $t$ and equals 0 otherwise). The per-period utilities $u_{k}\left(S_{t}\right)$ depend on the value of the vector of state variables $S_{t}$ during period $t$.

- Changes over time in the state variable $S_{t}$ are described by the Markov conditional density function

$$
f\left(S_{t} \mid S_{t-1}, D_{t}\right) .
$$

- Subject to regularity conditions, the maximum attainable utility of the infinite horizon maximization problem (17) can be recursively defined via Bellman's equation,

$$
W(S)=\max _{k}\left\{u_{k}(S)+\beta E_{S^{\prime} \mid S, k} W\left(S^{\prime}\right)\right\},
$$

where $W(S)=\max _{\left\{D_{t}\right\}_{t=1}^{\infty}} E \sum_{t=1}^{\infty} \beta^{t-1} \sum_{k=1}^{K} u_{k}\left(S_{t}\right) D_{t}$, given $S_{0}=S$. 
In usual circumstances, one would solve the dynamic programming problem by solving the functional equation (18) over a discretized state space, but if $K$ is large, then the dimensionality of the state space (more specifically, the number of "grid points" of the discretization) quickly becomes unwieldy. This quick increase of the state space dimensionality as the number of choices $K$ increases is often called the curse of dimensionality in DCDP problems.

For the matching model, one point in the state space consists of $\left(p^{1}, p^{2}, p^{3}\right.$, $\left.p^{4}, p^{5}, h\right)$, where $p^{j}, j=1, \ldots, 5$, is a probability that a patient places on drug $j$ 's being "high" quality and $h$ is the current recovery probability for the patient.

Keane and Wolpin (1994) develop an iterative method that overcomes this curse of dimensionality by solving the dynamic programming problem at a small set of points and interpolating over the other points.

\section{Adaptation of Keane-Wolpin method}

Fix a number of points in the state space; call this set of points $\tilde{S}$. Start with an initial guess of $W(S)$, at each point $S \in \tilde{S}$; call this initial guess $W^{0}(S)$.

Now we posit a relationship between each point $W(S)$ and the elements in the state space $S$. In the current specification, we assume that $W(S)$ is linear in $g(S)$, an $l$-dimensional (where $l$ need not equal $k$ ) vector of functions of the elements of $S$; i.e.,

$$
W\left(S_{t}\right)=g\left(S_{t}\right)^{\prime} \Gamma+\iota_{t}, \quad \forall S_{t} \in \tilde{S},
$$

where $S_{t}$ includes a constant term, $\Gamma$ is an $l$-dimensional vector of linear coefficients, and $\iota_{t}$ is a mean-zero error term, i.i.d. over $t$.

For the state points in $\tilde{S}$, therefore, we run the linear regression (19), to obtain coefficient estimates for $\Gamma$, which we denote $\Gamma^{0}$ (since these correspond to the zeroth iteration $W^{0}$ of the value function). Based on $\Gamma^{0}$, we approximate $W^{0}(S)$ for points $S \notin \tilde{S}$ by $W(S)=S^{\prime} \Gamma^{0}$.

Next we iterate the above process. First, for all the points $S \in \tilde{S}$, we construct the first iteration of the value function,

$$
W^{1}(S)=\max _{k}\left\{u_{k}(S)+\beta E_{S^{\prime} \mid S, k} W^{0}\left(S^{\prime}\right)\right\}
$$

where, for all required points $S^{\prime} \notin \tilde{S}$, we approximate using $W\left(S^{\prime}\right)=g\left(S^{\prime}\right)^{\prime} \Gamma^{0}$. Now, based on $W^{1}(S)$, we run a regression analogous to (19) in order to get $\Gamma^{1}$.

We stop this process at a given iteration $m$ if a uniform convergence criterion is satisfied:

$$
\max _{S \in \tilde{S}}\left|W^{m}(S)-W^{m-1}(S)\right|<\eta .
$$


After convergence, we use $W^{t}(S)$ as our value function for $S \in \tilde{S}$ and use $W(S)=g(S)^{\prime} \Gamma^{t}$ otherwise. $^{49}$

Each point is (componentwise) drawn randomly. The five discrete state variables $l_{j 1}^{m}, \ldots, l_{j 5}^{m}$ are drawn uniformly from the integers $0, \ldots, 10$. The five posterior means $\mu_{j 1}^{m}, \ldots, \mu_{j 5}^{m}$ are drawn randomly from a normal distribution with mean equal to the mean match value and variance equal to the initial variance. The recovery probability $h_{j t}^{m}$ is drawn from a unit uniform distribution. (The additional state variables are the logit error terms, which are explicitly integrated over in deriving the likelihood function.)

\section{REFERENCES}

ACKerberg, D. (2003): "Advertising, Learning, and Consumer Choice in Experience Good Markets: A Structural Examination," International Economic Review, 44, 1007-1040.

Ackerberg, D., AND M. RYSman (2003): "Unobserved Product Differentiation in Discrete Choice Models: Estimating Price Elasticities and Welfare Effects," RAND Journal of Economics, forthcoming.

ARNST, C. (2004): "The Waning of the Blockbuster Drug," Business Week, October 18.

Arrow, K. (1963): "Uncertainty and the Welfare Economics of Medical Care," American Economic Review, 53, 941-973.

BAILY, M. (1997): “Comments on Berndt, Cockburn, Griliches, 'Pharmaceutical Innovations and Market Dynamics: Tracking Effects on Price Indexes for Antidepressant Drugs', in Brookings Papers on Economic Activity: Microeconomics. Washington, DC: Brookings Institution.

Bergemann, D., AND J. VÄLIMÄKI (2004): “Monopoly Pricing of Experience Goods,” Working Paper, Yale University.

BlaU, D., AND D. GILlESKIE (2000): "A Dynamic Structural Model of Health Insurance and Retirement," Working Paper, University of North Carolina.

CAVEs, R., M. Whinston, AND M. HurWITZ (1991): "Patent Expiration, Entry and Competition in the US Pharmaceutical Industry," in Brookings Papers on Economic Activity: Microeconomics. Washington, DC: Brookings Institution.

Chernew, M., G. Gowrisankaran, AND D. ScAnlon (2004): "Learning and the Value of Information: The Case of Health Plan Report Cards," Unpublished Manuscript, University of Michigan.

CHING, A. (2002): "Dynamic Equilibrium in the US Prescription Drug Market after Patent Expiration," Unpublished Manuscript, University of Toronto.

Coscelli, A. (2000): “The Importance of Doctors' and Patients' Preferences in the Prescription Decision," Journal of Industrial Economics, 48, 349-369.

Coscelli, A., AND M. SHum (2004): “An Empirical Model of Learning and Patient Spillovers in New Drug Entry," Journal of Econometrics, 122, 213-246.

CURRIE, G., AND S. PARK (1999): "Estimating the Effects of Marketing and Consumption Experience on Demand for Antidepressant Drugs," Unpublished Manuscript, SUNY-Stony Brook.

DeGroot, M. (1970): Optimal Statistical Decisions. New York: McGraw-Hill.

ECKSTEIN, Z., D. HoRSKY, AND Y. RABAN (1988): "An Empirical Dynamic Model of Optimal Brand Choice," Working Paper, Tel-Aviv University.

\footnotetext{
${ }^{49}$ In practice, since the state space is continuous, we will never be called upon to calculate $W(\cdot)$ for exactly $S \in \tilde{S}$, so we will approximate $W$ at all points $S$. For our results, we use 1,012 points for the 11-dimensional state space.
} 
ECKSTEIN, Z., AND K. WOLPIN (1987): "On the Estimation of Labour Force Participation, Job Search, and Job Matching Models Using Panel Data," in Advances in the Theory and Measurement of Unemployment, ed. by Y. Weiss and G. Fishelson. New York: MacMillan, 82-112.

Ellison, S., I. COCKBURN, Z. GRILICHES, AND J. HAUSMAN (1997): "Characteristics of Demand for Pharmaceutical Products: An Examination of Four Cephalosporins," RAND Journal of Economics, 28, 426-446.

ERdeM, T., AND M. KeANE (1996): "Decision-Making under Uncertainty: Capturing Dynamic Brand Choice Processes in Turbulent Consumer Goods Markets," Marketing Science, 15, 1-20.

Ferreyra, M. (1999): "Physicians' Learning about New Drugs: An Empirical Approach," Unpublished Manuscript, Carnegie-Mellon University.

FRANK, R., AND D. SALKEVER (1997): "Generic Entry and the Pricing of Pharmaceuticals," Journal of Economics and Management Strategy, 6, 75-90.

GILlESKIE, D. (1998): "A Dynamic Stochastic Model of Medical Care Use and Work Absence," Econometrica, 66, 1-45.

Gourieroux, C., AND A. Monfort (1996): Simulation-Based Econometric Methods. London: Oxford University Press.

GRABOWSKI, H., AND J. VERNON (1992): "Brand Loyalty, Entry, and Price Competition in Pharmaceuticals after the 1984 Drug Act," Journal of Law and Economics, 35, 331-350.

HeCKMAN, J., AND B. Singer (1984): "A Method for Minimizing the Impact of Distributional Assumptions in Econometric Models for Duration Data," Econometrica, 52, 271-320.

HellersteIn, J. (1997): "The Importance of the Physician in the Generic versus Trade-Name Decision," RAND Journal of Economics, 27, 108-136.

HotZ, J., AND R. Miller (1993): "Conditional Choice Probabilities and the Estimation of Dynamic Models," Review of Economic Studies, 60, 497-529.

IIZUKA, T. (2004): "Experts Agency Problems: Evidence from the Prescription Drug Market in Japan," Working Paper, Owen Graduate School of Management, Vanderbilt University.

JOVANOVIC, B. (1979): "Job Matching and the Theory of Turnover," Journal of Political Economy, 87, 972-990.

JUDD, K. (1998): Numerical Methods in Economics. Cambridge, MA: MIT Press.

KEANE, M., AND K. WolPIN (1994): "The Solution and Estimation of Discrete Choice Dynamic Programming Models by Simulation and Interpolation: Monte Carlo Evidence," Review of Economics and Statistics, 76, 648-672.

KIHLSTROM, R., AND M. RIORDAN (1984): “Advertising as a Signal,” Journal of Political Economy, 92, 427-450.

LEE, L.-F. (1992): "On Efficiency of Methods of Simulated Moments and Maximum Simulated Likelihood Estimation of Discrete Response Models," Econometric Theory, 8, 518-552.

(1995): "Asymptotic Bias in Simulated Maximum Likelihood Estimation of Discrete Choice Models," Econometric Theory, 11, 437-483.

Milgrom, P., AND J. RoberTs (1986): "Price and Advertising Signals of Product Quality," Journal of Political Economy, 94, 796-821.

MiLler, R. (1984): "Job Matching and Occupational Choice," Journal of Political Economy, 92, $1086-1120$.

MirA, P. (2000): “Uncertain Child Mortality, Learning and Life-Cycle Fertility,” Unpublished Manuscript, CEMFI.

MotT, D., AND D. KRELING (1998): “The Association of Insurance Type with Costs of Dispensed Drugs," Inquiry, 35, 23-35.

MotT, D., J. SchOMmer, W. Doucette, AND D. Kreling (1998): "Agency Theory, Drug Formularies, and Drug Product Selection: Implications for Public Policy," Journal of Public Policy and Marketing, 17, 287-295.

Nelson, P. (1970): "Information and Consumer Behavior," Journal of Political Economy, 51, 311-329.

PAKeS, A., AND D. Pollard (1989): "Simulation and the Asymptotics of Optimization Estimators," Econometrica, 57, 1027-1057. 
Reuters (2004): "Britain Launches Study into Personalized Medicine," Reuters, September 19. RusT, J. (1987): "Optimal Replacement of GMC Bus Engines: An Empirical Model of Harold Zurcher," Econometrica, 55, 999-1033.

(1996): "Numerical Dynamic Programming in Economics," in Handbook of Computational Economics, ed. by D. K. H. Amman and J. Rust. Amsterdam: North-Holland, Chap. 14. ScOTT-MorTon, F. (1999): "Entry Decisions in the Generic Drug Industry," RAND Journal of Economics, 30, 421-440.

SHAPIRO, C. (1983): “Optimal Pricing of Experience Goods," Bell Journal of Economics, 14, 497-507.

STERN, S. (1996): “The Demand for Pharmaceuticals,” Unpublished Manuscript, Kellogg School. STERn, S., AND M. TRAJTEnberg (1998): "Empirical Implications of Physician Authority in Pharmaceutical Decisionmaking," Working Paper 6851, NBER.

Temin, P. (1980): Taking Your Medicine: Drug Regulation in the United States. Cambridge, MA: Harvard University Press.

YAMADA, T. (1995): Textbook of Gastroenterology. New York: Lippincott-Raven. 